\title{
SCALES AND SCALE-LIKE STRUCTURES
}

\author{
A Dissertation \\ by \\ ERIC BENJAMIN LANDRENEAU
}

\begin{abstract}
Submitted to the Office of Graduate Studies of Texas A\&M University in partial fulfillment of the requirements for the degree of

DOCTOR OF PHILOSOPHY
\end{abstract}

May 2011

Major Subject: Computer Science 


\title{
SCALES AND SCALE-LIKE STRUCTURES
}

\author{
A Dissertation \\ by \\ ERIC BENJAMIN LANDRENEAU
}

\begin{abstract}
Submitted to the Office of Graduate Studies of Texas A\&M University in partial fulfillment of the requirements for the degree of

DOCTOR OF PHILOSOPHY
\end{abstract}

Approved by:

Chair of Committee, Scott Schaefer

Committee Members, Ergun Akleman

Jinxiang Chai

John Keyser

James Wall

Head of Department, Valerie E. Taylor

May 2011

Major Subject: Computer Science 


\begin{abstract}
Scales and Scale-like Structures. (May 2011)

Eric Benjamin Landreneau, B.S.; M.S., Texas A\&M University

Chair of Advisory Committee: Dr. Scott Schaefer
\end{abstract}

Scales are a visually striking feature that grows on many animals. These small, rigid plates embedded in the skin form an integral part of our description of fish and reptiles, some plants, and many extinct animals. Scales exist in many shapes and sizes, and serve as protection, camouflage, and plumage for animals. The variety of scales and the animals they grow from pose an interesting problem in the field of Computer Graphics.

This dissertation presents a method for generating scales and scale-like structures on a polygonal mesh through surface replacement. A triangular mesh was covered with scales and one or more proxy-models were used as the scales shape. A user began scale generation by drawing a lateral line on the model to control the distribution and orientation of scales on the surface. Next, a vector field was created over the surface to control an anisotropic Voronoi tessellation, which represents the region occupied by each scale. Then these regions were replaced by cutting the proxy model to match the boundary of the Voronoi region and deform the cut model onto the surface. The final result is a fully connected 2-manifold that is suitable for subsequent post-processing applications, like surface subdivision. 


\section{ACKNOWLEDGMENTS}

I would like to thank my family and friends for their support throughout my school years. The process has been long but rewarding.

Thanks also go to my committee members, Dr. Wall, Dr. Keyser, Dr. Chai, and Dr. Akleman, for their encouragement. My committee chair, Dr. Schaefer, has been an invaluable source of support and guidance. 
TABLE OF CONTENTS

CHAPTER

INTRODUCTION . . . . . . . . . . . . . . . . . . . . . 1

1. Goals ..................... . . 2

II DEVELOPMENT AND APPLICATIONS OF SCALES . . . . . 4

A. Biological History . . . . . . . . . . . . . . . . . 4

1. Fish . . . . . . . . . . . . . . . 5

a. Ostracoderms .............. . . 6

b. Acanthodians .............. . . 6

c. Placoderms . . . . . . . . . . . . . . . . 7

d. Chondrichthyes ............ . . . 7

e. Osteichthyes . . . . . . . . . . . . . 8

f. Sarcopterygii . . . . . . . . . . . . 9 9

2. Amphibians . . . . . . . . . . . . . 9

3. Reptiles . . . . . . . . . . . . . . . . . . . 10

4. Birds . . . . . . . . . . . . . . . 11

5. Mammals . . . . . . . . . . . . . . . . 12

6. Plants ................... . . 12

B. Artistic History . . . . . . . . . . . . . . . . . 14

1. Traditional Artwork . . . . . . . . . . . . . 14

a. Fish . . . . . . . . . . . . . . 15

b. Reptiles . . . . . . . . . . . . 16

c. Modern Art . . . . . . . . . . . . . . . . 17

d. Computer Graphics Artwork . . . . . . . . . . 18

C. Military History . . . . . . . . . . . . . . . . . . . . . . . . 19

III PREVIOUS AND RELATED WORK . . . . . . . . . . . . 22

A. Biological Phenomena in Computer Graphics . . . . . . . . 22

1. Plants .................... . . 23

a. Trees . . . . . . . . . . . . 23

b. Leaves . . . . . . . . . . . . . . . . . 23

2. Animals . . . . . . . . . . . . . . . 24

a. Skin . . . . . . . . . . . . . . 25

b. Hair . . . . . . . . . . . . . 25 
c. Feathers . . . . . . . . . . . 26

d. The Retina of the Eye . . . . . . . . . . 26

B. Surface Patterns for Computer Graphics . . . . . . . . . 27

1. Surface Geometry . . . . . . . . . . . . . . . 27

2. Pattern Synthesis . . . . . . . . . . 28

IV METHODOLOGY ..................... 30

A. Scales: Form and Function . . . . . . . . . . . . . . 30

1. Squamation ................ . . 31

a. Ordered Squamations . . . . . . . . . . . 31

b. Disordered Squamations . . . . . . . . . . . . . 32

c. Squamation Synthesis . . . . . . . . . . . 33

2. Symmetry . . . . . . . . . . . . . 33

a. Biological Symmetry . . . . . . . . . . . 33

b. Repetitive Parts . . . . . . . . . . . . 34

3. Imbrication . . . . . . . . . . . . . . . . . . . . . . . . . . . . 35

4. Orientation ............... . . 36

V SCALE CREATION THROUGH SURFACE REPLACEMENT 38

A. Squamation . . . . . . . . . . . . . . . . . . . . . . . . . 38

1. Voronoi Tessellation Creation . . . . . . . . . . 39

2. Lateral Line . . . . . . . . . . . . . . . . . . 42

3. Surface Vector Field . . . . . . . . . . . . . . . . 46

4. Scale Region Improvement . . . . . . . . . . . . . . . . 48

B. Scale Synthesis . . . . . . . . . . . . . . 50

1. Cutting ................... 52

2. Merging ............... . . 54

C. Summary . . . . . . . . . . . . 56

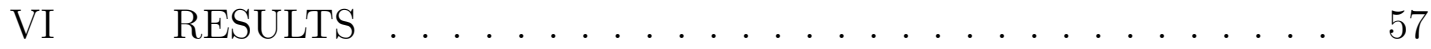

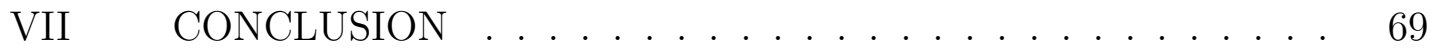

A. Contribution . . . . . . . . . . . . . . 69

B. Future Work . . . . . . . . . . . . . . . 71

1. Scale Shape. . . . . . . . . . . . 72

2. Scale Arrangement . . . . . . . . . . . . . . 72

3. Texturing and Shading . . . . . . . . . . 73

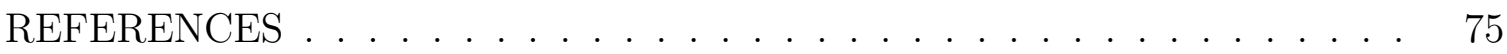


CHAPTER

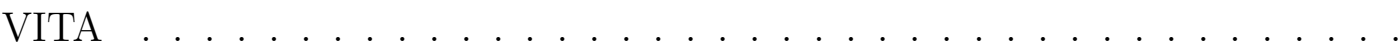




\section{LIST OF FIGURES}

FIGURE

1 Example of a cartilaginous fish, a white tip reef shark. Note that its placoid scales are extremely small, and only visible upon close inspection of the skin. Photo Credit:US National Oceanic and Atmospheric Administration [NOAA 2011]. . . . . . . . . . . .

Three examples of bony fishes. From left to right, a war-mouth bass, American plaice, and rainbow trout. Note how the number of scales may vary significantly depending upon species. Image credits: drawings by H.L. Todd, NOAA Photo Library [NOAA 2011

3 Reptile scales come in a variety of shapes. Left - A black-headed python is covered in smooth, flat scales. Right - A rattlesnake covered in keeled, or ridged, scales. . . . . . . . . . . . . .

4 Left - A pangolin, the only mammal possessing scales. Right - An armadillo, another armored mammal. Note the hexagonal scalelike pattern on the anterior and posterior scutes. Image credit: Jerry Segraves [Segraves 2007]. . . . . . . . . . . . . .

5 Shown above are several species of Madagascar plants covered in scale-like arrangements of thorns, on display at the California Academy of Sciences. From left to right - Alluaudia montagnacii, Alluaudia ascendens, Alluaudia procera, Didierea madagascariensis. .

6 A Chinese ink illustration of dragons covered in scales. From The Nine Dragons by Chen Rong, ca. 1244 AD [Rong 1244]. . . . . . . .

7 Examples of scale armor. Left - Gold scale parade armor worn by a Scythian royal. The armor consists of overlapping gold scales. Photo credit: ldot [Ldot 2007] Middle - Depiction of a battle against Sarmatians wearing scale armor. From Trajan's Column [Cichorius 1896]. Right - Roman scale armor, called "Lorica Squamata". Photo credit: Gaius Cornelius [Cornelius 2005]. . . . . 
FIGURE

8 X-ray of Dragon Skin armor. Ceramic bullet-proof plates overlap in a scale-like pattern to provide flexible protection. Image source: PEO Soldier [U.S. Army 2010]. . . . . . . . . . . . . . . . .

$9 \quad$ Illustration of hexagonal tiling, and the three principal directions of its dual. Kardong's work[Kardong 1998] notes these directions appear on many fish. . . . . . . . . . . . . . . . . .

10 Example of disordered squamation. The granular scales of anole lizards grow in a pattern resembling a blue noise distribution. . . . .

11 Left - a white ruffle sponge, an amorphous animal exhibiting very little symmetry. Middle - a starfish, showing radial symmetry. Right - a Caribbean spiny lobster, exhibiting bilateral symmetry. Also note the repetitive symmetry of its 6 legs and tail plates. Photo credits: NOAA Photo Library [NOAA 2011]. . . . . . . . . .

12 Left - an optimal circle packing, without overlap. Notice the unavoidable gaps between circles. Right - Imbrication of circles removes gaps, providing full coverage of the surface. . . . . . . .

13 Faces split and color according to three combinations of vertex colors - one-color (Top), two-color (Middle), and three-color (Bottom $) \ldots \ldots \ldots \ldots \ldots \ldots \ldots \ldots \ldots$

14 An illustration of Leucichthys sisco huronius, a Lake Huron herring. Red arrows denote the begin and end of the lateral line, which is highlighted to improve clarity. Image Credit: Charles B. Hudson, NOAA Photo Library [NOAA 2011]. . . . . . . . . . . .

15 Scale-sites spawn in 6 principal directions. Arrows in red denote scale-site orientations, and blue arrows represent spawning directions. . . . . . . . . . . . . . . . . . 
FIGURE

16 The user draws a lateral line (Top) to create several scale-sites along that line, colored dark, that we use to recursively spawn new scale-sites to fill the rest of the surface (Lower Left). After running Lloyd's algorithm with the scale-sites along the lateral line fixed in place, the sites converge (Lower Right). The arrows depict the orientation of each site and show how the lateral line influences the vector field.

17 We represent Voronoi sites as line segments, which can be adjusted to provide an isotropic tessellation (left, $\alpha=0$ ) or anisotropic tessellation (right, $\alpha>0$ ). Arrows denote the orientation of the site. . . . . . . . . . . . . . . . . .

18 Illustration of the homotopic relationship between a hexagonal tiling and rhombic tiling's face centers. . . . . . . . . . . . . . . . . 48

19 Voronoi tessellation on a model. We tessellate the surface of a model into Voronoi regions, where each region represents a single scale. . . . . . . . . . . . . . . . . .

20 Examples of different artist-created proxy models. . . . . . . . . . 50

21 Scale cutting, before(left) and after(right). A cutting boundary (red) derived from the scale's Voronoi region resizes until it fits the safety region(blue). . . . . . . . . . . . . . . .

22 Top left:triangulation of a proxy model with the boundary of the flattened Voronoi region in red. Top right: stellating the proxy model triangles using the boundary vertices. Bottom left: performing edge splits. Bottom right: shrinking the triangulation and stitching to the boundary. . . . . . . . . . . . . . . .

23 Above and below right - Bent cylinders with two types of scales applied. Above and below left - The pointed and rounded scales applied to the cylinders. 
25 A fish covered with 3306 scales using our technique. The scale generation region is blue-colored (top left). The user controls the distribution and orientation of the scales by drawing a lateral line on the fish. Our technique uses these inputs to generate a watertight model covered with scales (top right). The closeup (bottom middle) illustrates the detail achieved by our method. . . . . . . . .

26 Left - an illustration of a flounder (drawing credit: H.L. Todd, NOAA Photo Library [NOAA 2011]) Right - A flounder with ctenoid scales applied. Bottom - a closeup of the flounder's scales.

$27 \quad$ Scales applied to the extinct ostracoderm fish Drepanaspis. Two proxy models were used, for the backbone along a lateral line (upper left) and for the rest of the body (lower left). . . . . . . .

28 A lizard model with a lateral line painted down its back. Scale sites on the lateral line use a smooth spine for the proxy model(upper left), and the other sites use a barbed spine (lower left). The resulting surface smoothly joins both types of scales together. . . . . .

$29 \quad$ Lizard head with block-shaped scale (left) applied. . . . . . . . . . 61

30 A test of scales growing inward, under the surface, rather than outward. Left - the proxy model used to create the surface. . . . . .

31 Left - A face with a four-pronged scale applied. Right - the same model with Loop subdivision applied. Model credit: Jessica Riewe. . . . . . . . . . . . . . . . . . . .

32 Left A stegosaurus model, without back plates or tail spikes. The back plate growth region is colored green, and the tail spike region is colored red. Middle - proxy models for the plates and spikes. Right - the stegosaurus after plates and spikes have been added.

33 A dragon with three types of scales applied to three different regions - the back, flank, and head. . . . . . . . . . . .

$34 \quad$ A1, B3 - photographs of a Didiera madagascariensis and Alluaudia montagnacii, respectively. A2, B1 - CVTs from which thornscales were grown. $A 3, B 2$ - meshes of the plants with thorn-scales applied. 
FIGURE

35 Left - A dragon egg, covered in scales. Right - the same model, after surface simplification has been performed. . . . . . . . . . 66

36 A scale armored hauberk, showing scales generated by our technique. . . . . . . . . . . . . . . . . . . .

37 A problem case for our method. High frequency details and regions of high negative curvature with respect to the scale size may cause self-intersection or twisting during scale deformation.

38 A torus with teapot-shaped scales applied. Our method is robust enough to handle unusual shapes such as these. . . . . . . . . . . . 


\section{CHAPTER I}

\section{INTRODUCTION}

Scales are a visually striking feature that grows on many animals. These small, rigid plates embedded in the skin form an integral part of our description of fish and reptiles, some plants, and many extinct animals. Scales exist in many shapes and sizes, and serve as protection, camouflage, and plumage for animals. The variety of scales and the animals they grow from pose an interesting problem in the field of Computer Graphics.

Creation of 3D models for graphics purposes often involves representing shapes as surfaces of triangles. It is important that these surfaces be closed, as many subdivision, simplification, and shadowing algorithms require a closed manifold surface. As artists create more detailed surfaces, they are expected to preserve this closed property. Some forms of surface details can prove difficult or tedious to model. One such detail is the scale.

Scales overlap, have complex surfaces, and embed in the skin tissue of animals. Overlapping shapes cause problems for artists, as does embedding shapes into surfaces. In the case of scales, dealing with thousands of complex overlapping shapes can be quite problematic.

While scales are difficult to create, their creation is necessary due to their prominence in nature. Scales are widespread in the animal kingdom, occurring on a variety of animals. As external features that are easily visible, scales are commonly incorporated into 3D artwork in simple approximations as textures and displacement maps.

This dissertation follows the style of ACM Transactions on Graphics. 
However, despite their extensive use, there is little formal exploration the creation of scales for 3D meshes. There is a large body of work for related features such as skin, hair, and feathers, so an exploration of scales is justifiably needed for a comprehensive representation of animals in Computer Graphics.

Artists typically approach creation of scales by first creating an underlying, smooth mesh. This surface is then either covered manually with hundreds to thousands of small scales, or painted with a repetitive texture approximating a scaly surface. These scales must conform to the underlying surface, can overlap each other, and each scale may individually have a detailed shape with barbs or ridges. This application process can involve a great deal of effort and time, and gives us sufficient motivation to automate this process.

\section{Goals}

This dissertation describes a method of creating self-similar, overlapping scales on a mesh based on artistic guidance. We will present a novel technique for integrating scales onto a triangle surface which addresses the shape, orientation, and placement of the scales. This integration will form a watertight, geometrically 2-manifold surface suitable for subdivision and simplification. Producing a manifold shape is important for reasons beyond Computer Graphics as well. A manifold surface is necessary for creating many forms of sculptures, including recent scale-like shapes created by Peter Randall-Page [Randall-Page 2006] and Simon Thomas [Thomas 2006].

In the ensuing chapters, we first motivate scale generation by exploring the many forms and functions of scales. From this variety of scales, we observe common properties of scales and use them as a groundwork for our scale synthesis technique. There is scarce literature on scale synthesis for 3D graphics, so we adapt concepts from related graphics fields towards developing our scales. We create scale geometry on a variety 
of meshes, showing that it works well across a variety of shapes and configurations. We create scale geometry by segmenting the surface of a mesh into many singlescale regions. We replace each of these regions with a model of a scale which is cut and deformed to fit the region exactly and connect to neighboring scales. The work not only provides a way to create plausible and interesting scales on surfaces, but also gives inspiration into creation of new and unusual surface geometry. 


\section{CHAPTER II}

\section{DEVELOPMENT AND APPLICATIONS OF SCALES}

Scales have a variety of uses. They first evolved on animals millions of years ago as a form of flexible armor. Humans adapted scales for many uses, both artistic and practical. Scales continue to be utilized in a variety of formats today, and representing them in Computer Graphics proves an interesting challenge.

\section{A. Biological History}

Approximately 500 million years ago, many forms of advanced invertebrate life such as arthropods existed in Earth's oceans. Arthropods at the time, such as trilobites and sea scorpions, had no internal skeleton, instead relying on a hard, chitinous exoskeleton to support and protect their bodies.

Arthropod exoskeletons form large rigid regions, restricting movement to joints connecting regions together. An animal with an exoskeleton is not only restricted to movements based on its exoskeleton's segmentation, but vulnerable to attack at the joints between segments. Damage to an exoskeletal segment might crack the entire segment, harming or leaving vulnerable large areas of the animal's body.

During this time of arthropod domination of the oceans in the Cambrian Period, a new form of life appeared in the form of vertebrates. These vertebrates now had endoskeletons, in contrast with the external skeletons of arthropods. With the internalization of vertebrate's skeletons, they could no longer benefit from the armored protection enjoyed by arthropod. Instead, many vertebrates evolved an alternate defense mechanism - the scale.

Scales provided a new type of protection for organisms. Instead of surrounding an animal in large, rigid segments, scales cover an organism in a network of small, 
armored plates. Being smaller, the scales form a flexible sheetlike surface that is both resistant to attack and flexible. Scales can overlap, forming an imbricated network which fully covers the animals without the weak points of exoskeleton joints. These advantages make scales a powerful evolutionary advantage, one that persists in many animals today.

An indirect consequence of scale development is paleontological classification. The process of fossilization of an organism's body often eliminates traces of soft tissue. Soft-bodied animals produce poor or nonexistent fossils, and many primitive organisms remain undiscovered due to having no fossil record. However, scales contain tough materials that fossilize well, meaning that scaled animals will have a far more robust fossil record than similar, unscaled animals. In fact, a great number of primitive animals are identified by their scales alone, as the scales are all that remains of their bodies after fossilization. That species can be differentiated by their scales also emphasizes a large diversity in the structure and composition of scales.

\section{Fish}

The first known appearance of scales begins with primitive fish. Beginning with the emergence of fish in oceans over 500 million years ago, the majority of fish species have grown some form of dermal scales. In addition to protective properties, scales provide fish with locomotive and in the case of lateral lines, sensory abilities. A fish's locomotion is aided by the shape of scales, which help create a laminar flow of water around the animal. There are many varieties of fish scales, dating back to many prehistoric fish with types of scales not seen today. 


\section{a. Ostracoderms}

Scales are a prominent feature of the first vertebrates, the ostracoderms. Ostracoderms are boneless, jawless fish that evolved during the late Cambrian Period, roughly 520 million years ago. The word ostracoderm comes from the Greek roots ostrako, meaning shell, and -derm, meaning skin. This "shell-skin" name is due to the bony scales covering their bodies[Radinsky 1987]. While some ostracoderms such as anaspids grow small scales on their heads, others like the osteostracans create large headshields, like primitive skulls. Ostracoderms survived until the late Devonian Period, when they and many other animal species disappeared in a mass extinction. Even at this early stage of evolutionary development, ostracoderms exhibit much diversity in their scales. One group of ostracoderms, the thelodonts, are classified specifically by their unique scales. The word thelodont means "nipple-tooth", describing the pointed appearance of their spiny scales. The tooth reference in the name is doubly apt because like teeth, thelodont scales have a hard outer coating of dentine.

\section{b. Acanthodians}

Acanthodians, also known as spiny sharks, are the first known vertebrates with true jaws, appearing in the late Silurian Period about 410 million years ago. These fish are somewhat similar in form to modern sharks, but unlike sharks they have tiny rhomboid scales. The acanthodians went extinct by the end of the Permian period, about 250 million years ago. 
c. Placoderms

Also appearing during the Silurian Period and dying out in the Devonian extinction are the placoderms. These fish, similar to ostracoderms, possess armored heads and thoraxes along with a posterior sometimes covered in scales. Unlike the ostracoderms, placoderms have true, moveable jaws.

d. Chondrichthyes

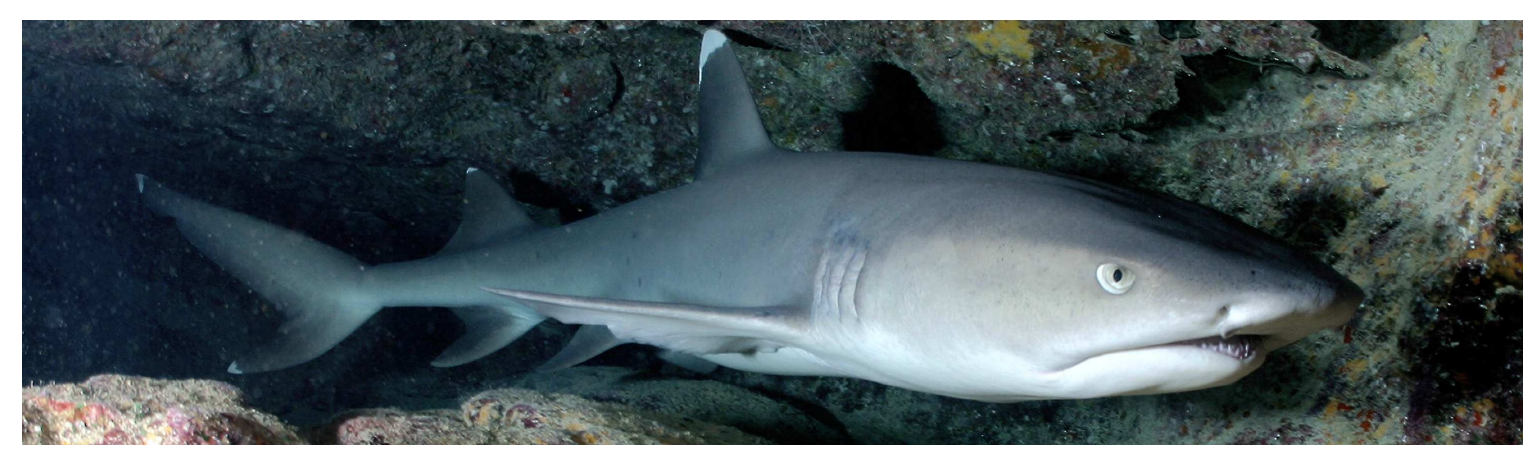

Fig. 1. Example of a cartilaginous fish, a white tip reef shark. Note that its placoid scales are extremely small, and only visible upon close inspection of the skin. Photo Credit:US National Oceanic and Atmospheric Administration [NOAA 2011].

Chondrichthyes, seen in Figure 1, are cartilage-skeletoned fishes evolved from placoderms roughly 395 million years ago in the Devonian Period. Many of these fish still exist today as sharks, rays and skates. These fish are covered in bony, small scales called denticles due to their similarity to teeth. Unlike the scales of bony fish, denticles do not grow with the fish. As chondrichthyes mature, new denticles grow in between old scales to fill in gaps created by body enlargement. 


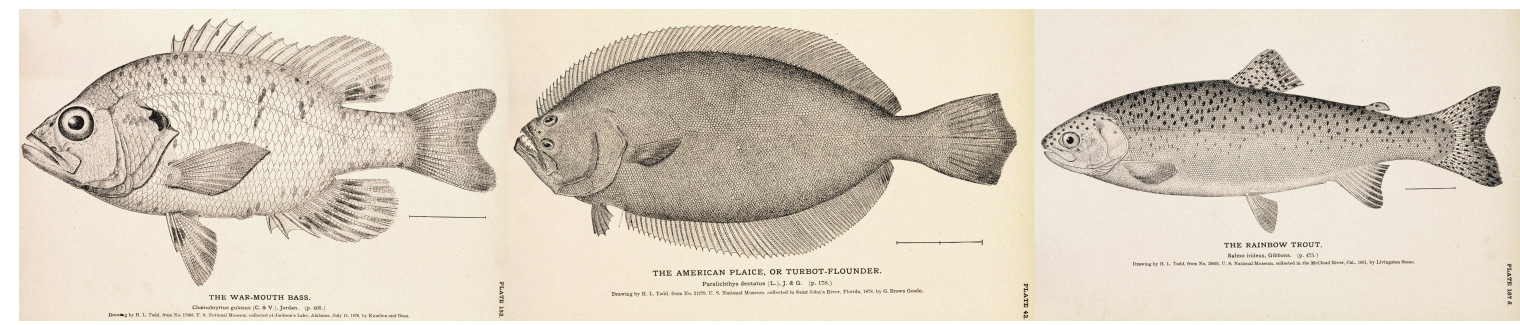

Fig. 2. Three examples of bony fishes. From left to right, a war-mouth bass, American plaice, and rainbow trout. Note how the number of scales may vary significantly depending upon species. Image credits: drawings by H.L. Todd, NOAA Photo Library [NOAA 2011].

\section{e. Osteichthyes}

Modern bony fish, called Osteichthyes, also evolved around the same time as the Chondrichthyes. These fish, illustrated in Figure 2, have a bony internal skeleton and comprise the majority of modern fishes. Osteichthyes possess many types of scales:

1. Cosmoid scales consist of four layers of bony material. True cosmoid scales existed on the now extinct Crossopterygian class of lobe-finned fish. Lungfish and coelacanths descended from the Crossopterygians and possess modified cosmoid scales.

2. Ganoid scales, generally rhombic in shape, are similar in structure to cosmoid scales but have a thicker layer of Ganoin. These scales are found on garfish, sturgeon, and related fish.

3. Leptoid scales are the most common form of fish scale. Also known as elasmoid scales, they contain much less bone and are more flexible than other types of scales. Leptoid scales form rings as they grow, much like the rings found in the trunks of trees. The age of a fish can be determined by counting the rings in its scales. 
(a) Cycloid scales are smooth-edged leptoid scales.

(b) Ctenoid scales exhibit rough, serrated edges.

It is worth noting that both cycloid and ctenoid scales may be present on a single fish, such as the flounder. The shape of individual scales may vary a great deal on an single fish, as illustrated in Patterson et al.'s recent atlas of 48 fish species [Patterson et al. 2002].

As Osteichthyes fish grow, so do their scales, unlike Chondrichthyes. In some cases, particularly the Japanese koi, the scales of these bony fish may grow faster than the fish, leading to scale shedding. Some species of bony fish such as herring have deciduous scales, meaning their scales easily fall off, usually seasonally or at certain points in the fish's life cycle.

\section{f. Sarcopterygii}

The Sarcopterygians, also known as lobe-finned fish, represent a pivotal moment in vertebrate evolution. All four-limbed vertebrates, called tetrapods, are descended from these fish. Amphibians, reptiles, dinosaurs, birds, and mammals are tetrapods. Sarcopterygians possessed cosmoid scales, as described previously in subsection e.

\section{Amphibians}

Amphibians were the first tetrapods, and named for their ability to live on land and in water. They evolved during the Devonian Period. The only amphibians to possess scales are caecilians, which grow calcite scales embedded in their skin. 

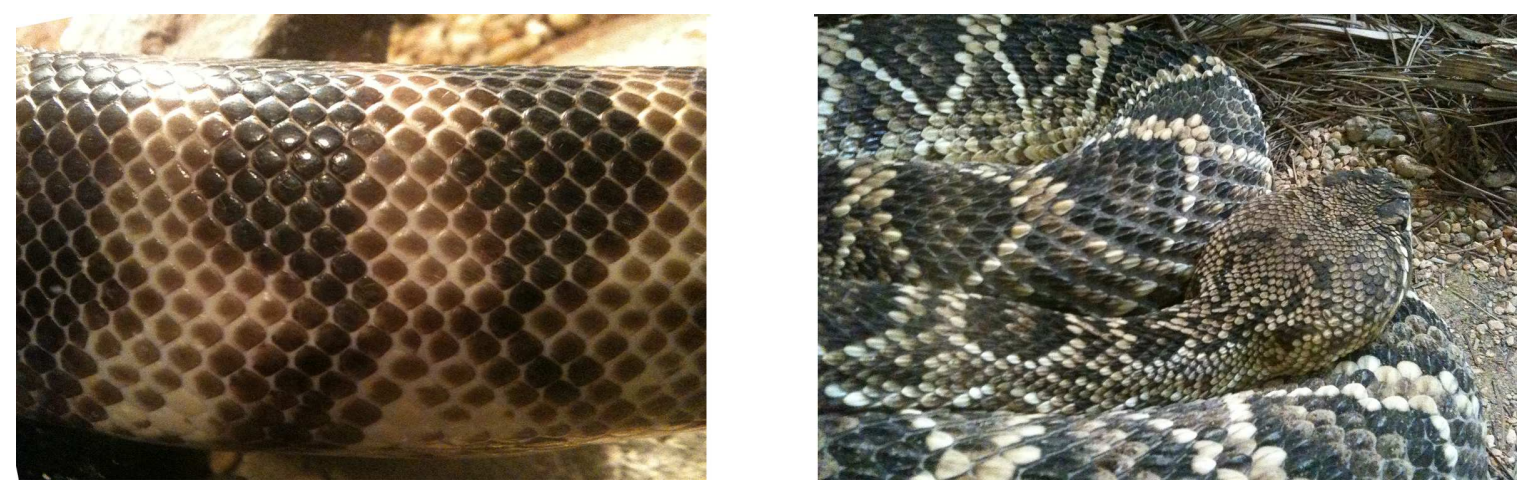

Fig. 3. Reptile scales come in a variety of shapes. Left - A black-headed python is covered in smooth, flat scales. Right - A rattlesnake covered in keeled, or ridged, scales.

\section{Reptiles}

During the late Carboniferous Period, about 310 million years ago, reptiles evolved from amphibian labyrinthodonts. Reptiles are notable for their scaly skin, a marked change from the mostly scaleless amphibians. Reptile scales are mostly composed of keratin and come in a large variety of shapes. Some features of reptile scales include:

1. Imbricate reptile scales overlap similarly to many fish scales.

2. Granular scales appear as bumps on the skin of the reptile.

3. Keeled scales exhibit a ridge down the center. Figure 3 shows an example of keeled scales on a rattlesnake.

4. Scutes are very large scales which may form bands across the animal such as crocodiles, or form carapaces in the case of tortoises.

Like fish, reptile scales vary in shape, size, and positioning. Reptile scales may be arranged in rows, as seen on the boa in Figure 3, form disorganized patterns, or a combination of both on the animal. While this variation in arrangement also exists in fish, the pattern variations formed by granular scales and the like are typically less 
ordered than any seen in fish.

The variety of reptile scales makes them a useful identifying characteristic for each species. Identification of scale patterns is particularly useful for identification of poisonous and nonpoisonous snakes. Nonpoisonous snakes have two rows of scales on their belly, while poisonous snakes have a single row of scales along most of their underside.

Reptile scales serve many additional purposes. They help in heat regulation, a useful feature for coldblooded animals. Scale coloration serves as useful camouflage, as well as warning colors which scare away predators. Scales are even used for locomotion in snakes.

\section{Birds}

Although there is still debate over the specific origin of class Aves, or birds, many paleontologists consider Archaeopteryx to be one of the first birds, as a transition from the reptilian dinosaurs during the Jurassic Period 150 million years ago. Birds possess thick scales on their feet called scutes. Interestingly, these scutes are chemically very similar to the large scales found on the bodies of crocodiles. In addition to the scutes, bird feet also have small scales, called reticulae, on the bottom of the digits of their feet.

Birds do not exhibit scales apart from those on their feet. However, it is interesting to note that bird feathers seem to be evolutionarily linked to reptile scales. Some research by Zou et al.[Zou and Niswander 1996] even shows that bird scutes can be induced to develop into feathers, suggesting a genetic correlation between a bird's scales and feathers. 


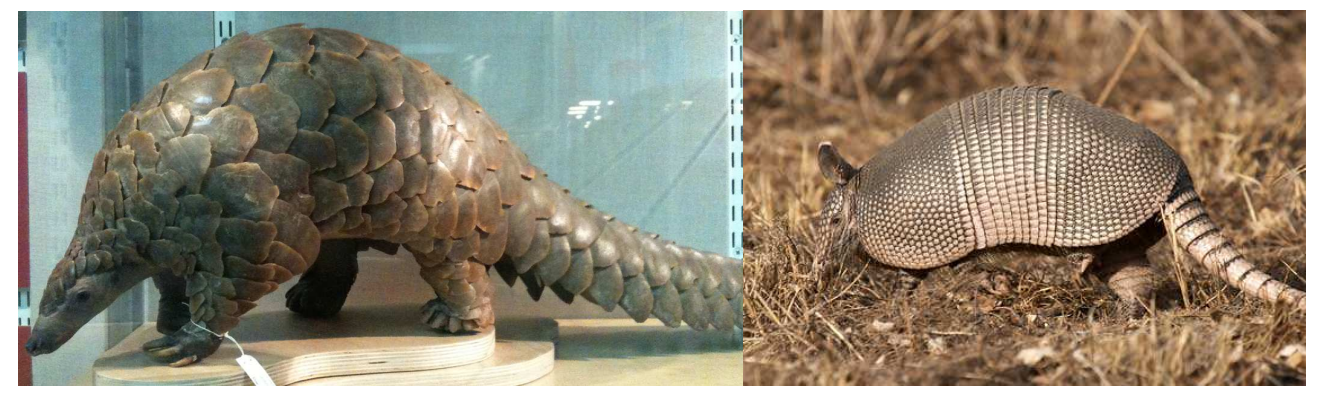

Fig. 4. Left - A pangolin, the only mammal possessing scales. Right - An armadillo, another armored mammal. Note the hexagonal scale-like pattern on the anterior and posterior scutes. Image credit: Jerry Segraves [Segraves 2007].

\section{Mammals}

The first mammals are believed to have appeared about 205 million years ago in the Triassic Period. There is one mammal possessing scales, the pangolin (Figure 4.) or scaly anteater. This unusual mammal's scales are actually modified hairs, made of keratin. Pangolin scales are particularly effective as body armor, as they can curl into a ball for defense and the scales have sharp edges in addition to being tough.

Although the pangolin is the only mammal with scales, some other mammals have scale-like structures. The spiny anteater, or echidna, is covered in spines. Other mammals possess spines, including the hedgehog and porcupine. Another armored mammal, the armadillo (Figure 4), is covered in bony scale-like scutes. The armadillo's scutes also have hexagonal patterns of bumps which appear similar to the granular scales of reptiles.

\section{Plants}

Animals are not the only organisms which grow scales. Scale features exist on many species of plant life. Even plant leaves have scale-like properties, being protective, overlapping, symmetric features emerging from the surface of the plant; Charles 
Breder notes this commonality in his study on organic symmetry [Breder 1947]. However, for simplicity's sake we will exclude leaves from consideration as scale-like features and focus only on external bark structures.

One prehistoric plant had features that earned it the nickname the "scale tree." The Lepidodendron, an ancient lycopod, is covered in diamond-shaped leaf scars on its midsection. Unlike animal scales, these marks are not formed by hardened subsurface plates, and are instead created by the plant growing and shedding leaves, leaving indentations in the bark corresponding to leaf stems. Many modern day palm trees exhibit similar features on their trunk, created by the upward shedding of leaves as the plant grows.
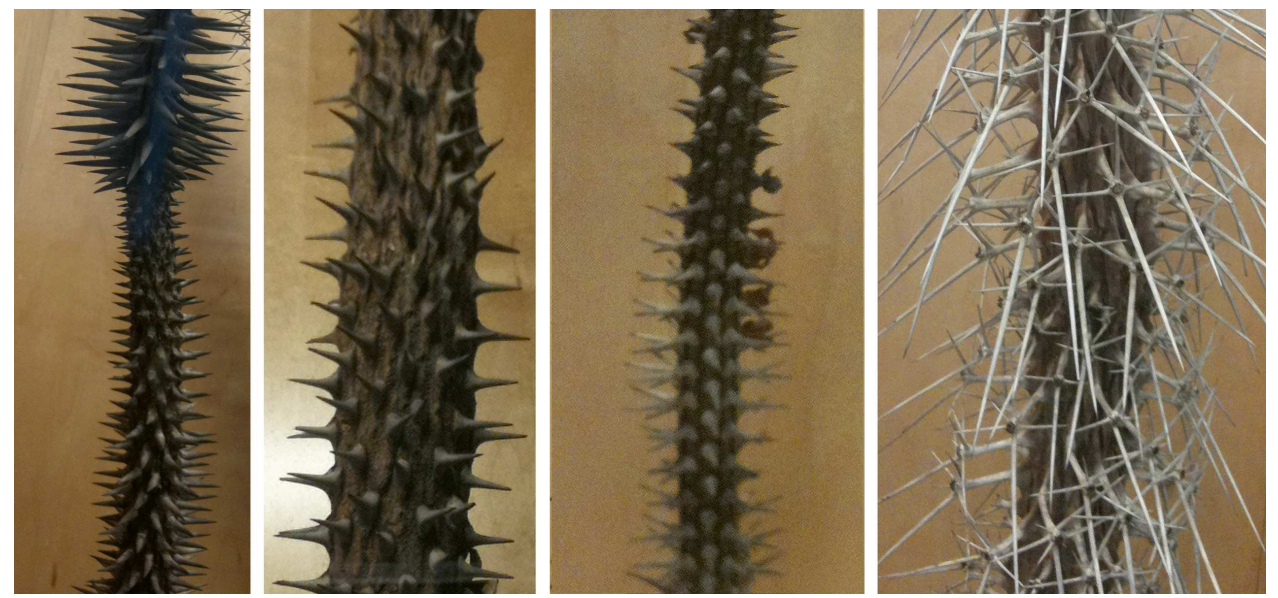

Fig. 5. Shown above are several species of Madagascar plants covered in scale-like arrangements of thorns, on display at the California Academy of Sciences. From left to right - Alluaudia montagnacii, Alluaudia ascendens, Alluaudia procera, Didierea madagascariensis.

Other plants form symmetric, scale-like structures on their branches and stems. Thorny plants such as roses exhibit scale-like properties in their thorns, although thorns are usually too widely spaced to be classified as scale structures. There do exist some plants with tightly packed thorns, in particular many species from Madagascar 
such as Alluaudia montagnacii and Alluaudia ascendens, shown in Figure 5. These tightly packed thorns bear many similarities to scales, with the primary difference being that their shapes are less flat and imbricating than typical scales. Instead of forming a hard, ablative surface, the thorns of these plants form a pointy, piercing surface.

We note at this time an interesting two-fold property of scales which will be discussed further in later chapters. This property is the description of scales by their arrangement on a surface, and by their local shape. The arrangement of scales, called the squamation, describes where scales are positioned on the surface, and the local shape describes the shape of a single scale at its position on the surface, as well as how it connects to neighboring scales. What is interesting about this is that the arrangement of the thorns on the aforementioned Madagascar species follows the same patterns as the arrangements of animal scales. Only the local shape of the thorns differs from animal scales.

\section{B. Artistic History}

\section{Traditional Artwork}

In addition to being a common biological feature, scales also feature prominently in artwork, as they provide a visually striking way to add detail to objects. Scales are depicted by many techniques including circular or crosshatched shapes for fish, granular or pointed scales for reptiles, and so on. Scales appear both directly on figures such as fish, animals and humanoid characters, and as patterns. Abstract tiled scale patterns also appear frequently, and are especially prominent on pottery throughout human history. 


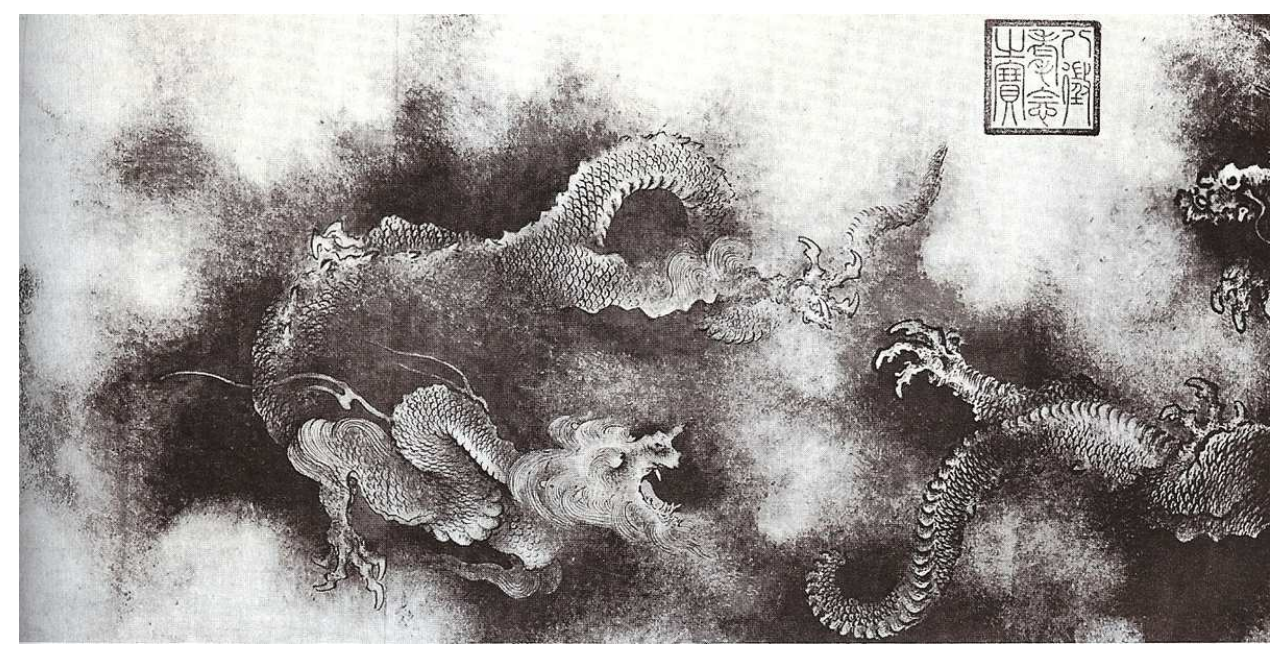

Fig. 6. A Chinese ink illustration of dragons covered in scales. From The Nine Dragons by Chen Rong, ca. 1244 AD [Rong 1244].

a. Fish

Fish hold significance in many human cultures, being an easily attainable and prolific food source. Fish scales often form striking, colorful patterns, so the representation of these scales in artwork is a natural transition. Many forms of art directly incorporate real fish scales into jewelry or clothing.

The fish is an important religious symbol, and is represented often in religious artwork. The ancient Philistine fertility god Dagon is depicted as a merman, with half his body fish-like and covered in scales. The Hindu god Vishnu's first incarnation, Matsya, is also represented as a merman or sometimes an entire fish. The merman or mermaid motif, often representing deities, also occurs in the artwork of the Assyrian, Babylonian, Sumerian and Phoenician cultures. The motif appears in many places in Greek mythology, representing Triton, Glaucus, Pan, and other gods. Traditional folklore of many other world cultures also depict merfolk as magical creatures.

In addition to religious importance, fish are revered in many Asiatic cultures and 
their artwork, particularly carp. Both Chinese and Japanese tradition consider the carp to represent luck, and in the Mandarin language the words "fish" and "abundance" are pronounced the same. Fish are prolific in east Asian artwork, with particular emphasis on their scales.

\section{b. Reptiles}

Reptiles also appear in many forms of art. Scaly reptilians often have significant mythological meanings. Snakes or serpents are represented in many different cultural mythologies and have many connotations, from representing knowledge and temptation in the Christian Bible, to creation stories in the Chinese, Egyptian and Greek cultures where a snake gives birth to humans and animals. Many other cultures incorporate serpent symbology into their beliefs. Serpent symbology varies considerably across cultures with representations of good, evil, and ambivalence, and are one of the most widespread, oldest mythological symbols. Many forms of religious artwork incorporate serpents, and most representations feature illustration of scales on the body of the serpent.

Tied to the depiction of serpents in artwork is images of scale-covered dragons. Many medieval European cultures directly correlated dragons and snakes, often mixing descriptions of the two. For example the Old English poem Beowulf describes a creature with both the word for dragon, draca, and the word for serpent, wyrm. Many European cultures interchanged descriptions of dragons and serpents, always referring to evil, scaled mythical beasts. Artwork of these creatures usually show aggressive forms with sharp features, with corresponding sharpness shown in the scales.

East Asian depictions of dragons, as seen in Figure 6, differ from Western dragons primarily by being representations of good rather than evil. Asian dragons appear as large, snakelike, scale-covered creatures similar to their European counterparts, but 
otherwise exhibit different symbolism. While the Western dragons are associated with evil, fire, poison, and treasure-hoarding, Asian dragons typically symbolize strength and luck. Dragon symbology pervades east Asian culture, with examples of artwork dating back to the Neolithic Yangshao culture in China over six thousand years ago. The scales of Asian dragons are said to represent the scales of a carp, and they are typically depicted in art as flat, circular shapes. The prominence of dragons and carp in East Asian artwork persists today.

\section{c. Modern Art}

In addition to scales appearing on animals in artwork, scales also occur in modern abstract and mathematical art, as well as in architecture. The symmetric, patterned nature of scales lends to the use of scales as textures and mosaics.

Because scales tend to grow closely together on animals, they form packing patterns. As the animal grows, this pattern may change through the introduction of new scales in cartilaginous fish (Section A, subsection 1d), or as the animal sheds scales. These squamations may be very ordered and form hexagonal or square patterns, or disordered such as those of skates and rays. These packing patterns, especially rhombic and hexagonal shapes, are often adapted to artistic tilings. Less organized patterns tend to form blue noise distributions, and can be represented through Voronoi tessellations.

Scale patterns, especially those based off cycloid scales (Section A, subsection 1e), are commonly seen in architectural designs. Many varieties of scale-based floor and wall tiles exist. Roof shingles are generally imbricated to weatherproof to avoid water pooling. Because most types of animal scales are also tileable, symmetric, and imbricated, using shingles based on fish scales is intuitive. The architectural work of Frank Gehry is particularly inspired by fish and their scales. 
One noted use of scales in a more abstract manner is in Islamic artwork. While fish and other animals occasionally appear, Islamic artwork historically focused on producing interesting geometric patterns. According to Denny [Denny 1974], during the sixteenth century in the Ottoman Empire there was a particular focus on imitating Chinese blue-and-white porcelain. This naturally led to imitation of geometric patterns present in Chinese artwork, especially scale patterns.

The popular Dutch artist M.C. Escher expressed a particular interest in scaly animals. Much of his art involves mathematically-inspired patterns of fish, lizards, and snakes. In particular, Escher's Fish and Scales depicts a recursive tessellation of fish evolving from the scales of a larger fish.

Some artwork incorporates actual animal scales. Many articles of clothing, in particular jackets, purses and boots, use scaly reptile skin for its attractive appearance. A particular culture in northern China, the Hezhe, makes garments out of fish skin. Even when actual animal scales are not incorporated into clothing, scale-like shapes are often integrated into clothing to make aesthetically pleasing patterns. Many types of jewelry also use artificial and natural scales. Popular fashion's widespread usage of scales attests to their visually pleasing nature.

\section{d. Computer Graphics Artwork}

Scales are often implemented in Computer Graphics, or CG, artwork, typically emerging as skin texturing for characters. A variety of scale-covered CG animals commonly appear in commercial art, including dragons, snakes, fish, dinosaurs, lizards, and many more. Many films include scaly animals as main characters, including dragons in Dragonheart, Reign of Fire, Harry Potter and the Goblet of Fire and How to Train Your Dragon, many dinosaurs in Jurassic Park and Ice Age 3, fish in Finding Nemo, and so on. 
The scales on these creatures are usually created by artists manually painting or modeling the scales. This process is painstaking, as a creature may be covered by thousands of scales. Painted scales are often turned into displacement maps to add additional shape to the scales. Larger scales may be modeled directly on the surface.

While there are a variety of artistic techniques for creating 3D scales, there has been little formal exploration of the subject. Most techniques are specifically designed for a single character, and not easily generalizable to other shapes.

\section{Military History}
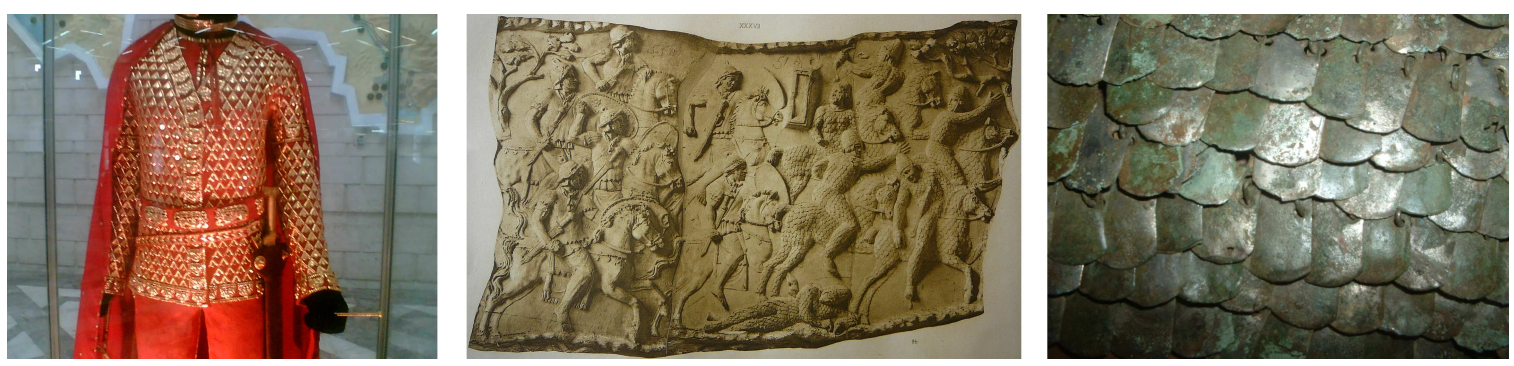

Fig. 7. Examples of scale armor. Left - Gold scale parade armor worn by a Scythian royal. The armor consists of overlapping gold scales. Photo credit: ldot [Ldot 2007] Middle - Depiction of a battle against Sarmatians wearing scale armor. From Trajan's Column [Cichorius 1896]. Right - Roman scale armor, called "Lorica Squamata". Photo credit: Gaius Cornelius [Cornelius 2005].

As scales form a natural layer of armor for animals, it is unsurprising that they prove useful in designing military armor. Armor covered in scales bends to conform to the body of a soldier, deflecting attacks without sacrificing mobility. The selfsimilar, tileable nature of scales also simplifies manufacture of the armor, as scales can be mass produced and fitted to any underlying shape. The individual scales are produced separately, then typically woven together and attached to an underlying armor. 
The use of scales in protective armor dates back at least to the ancient Persians. Herodotus also makes mention of Persian scaled armor, describing colored tunics covered in iron fish scales [Laufer 1914] [Herodotus 1954], and Persian Elamite artwork as early as the third millennium BCE depicts people wearing armor covered in scales.

During the first millennium BCE a nation of nomads, the Scythians, rose to prominence in the region of modern day Iran. The Scythians used many forms of scale armor to outfit their cavalry. They sewed iron and bronze scales onto leather armor which covered the neck to hips, eventually improving the armor to cover the shoulders and helmet. Scale armor was also adapted to fit horses. A related group of Iranian people during this era, the Sarmatians, were called "lizard people" for their distinctively reptilian scale armor. Figure 7 shows examples of Sarmatian and Scythian armors.

Scale armor remained prominent throughout the reign of the Roman Empire, providing an alternative to mail armor. Regular troops wore a variety of scale armor named lorica squamata (see Figure 7), while officers wore a hybrid mail covered in very small scales, called lorica plumata [Bishop and Coulston 1993]. In the Byzantine Empire, scale armor evolved into lamellar armor, formed from arrangements of small plates.

Armor made of scales remained popular long after the decline of the Roman Empire, finally falling out of fashion during the Middle Ages. Some forms of scale armor persisted elsewhere, such as the Japanese gyorin kozane worn by samurai (see Figure 7), and Indian pangolin-scale armor.

Although scale armour declined in popularity with the advent of modern warfare, using scales to provide flexible protection persists. One example of modern scale armor is Dragon Skin, seen in Figure 8, a ballistic vest produced by the Pinnacle Armor corporation. These vests contain an arrangement of ceramic plates designed 


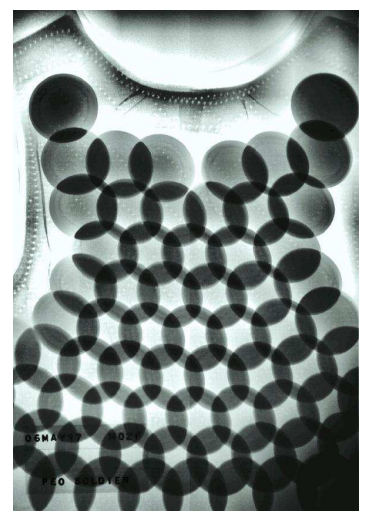

Fig. 8. X-ray of Dragon Skin armor. Ceramic bullet-proof plates overlap in a scale-like pattern to provide flexible protection. Image source: PEO Soldier [U.S. Army 2010]. to withstand small arms fire, and can reportedly provide protection against grenades. 


\section{CHAPTER III}

\section{PREVIOUS AND RELATED WORK}

Scale creation for Computer Graphics is a relatively unexplored field. Despite the constant usage of scales in a variety of CG media, there is little formal or detailed exploration of the subject matter. We seek to bridge this gap by applying concepts from related areas of CG graphics to our scale synthesis.

\section{A. Biological Phenomena in Computer Graphics}

While scales are a relatively unexplored topic in Computer Graphics, many other features of animals do. Many external features such as hair, feathers, or skin are shared by entire classes of vertebrate animals, and techniques for representing these features are invaluable to artists. Although the field of scale generation remains fairly small, we seek to apply CG modeling of other biological concepts towards that of scales.

Current representations of living organisms in CG focus on two kingdoms, animals and plants. One explanation for this focus is that plants and animals are the forms of life we are most familiar with. Other kingdoms such as fungi are often microscopic or amorphous and difficult to recognize, with mushrooms being the only form of fungus easily recognized by a layman. While there is some work in graphics visualization of fungi such as Meskkauskas et al.'s simulation of hyphal growth [Meskkauskas et al. 2004], most CG work involving bacteria and fungi focuses on analysis and identification, not generation. 


\section{Plants}

Representing botanical life is a popular topic in Computer Graphics. Plants, especially trees, provide people with food, raw construction materials, decoration, and much more. Plants play a significant role in peoples' lives, and as such they become an important element to represent in Computer Graphics.

\section{a. Trees}

Trees remain a significant area of Computer Graphics work, both for artistic and architectural purposes. Ulam [Ulam 1962] and Honda [HONDA 1971] developed seminal methods for generating tree shapes. Honda's work creates trees explicitly through recursive branching, whereas Ulam's develops a volumetric tree shape by selforganization of many units competing for space. Many different techniques for tree modeling now exist such as a recent interactive tree-generation method by Palubicki et al. Palubicki [Palubicki et al. 2009], and tree generation remains an active topic.

A subset of tree modeling which relates to our scale creation is bark synthesis. Bark of trees forms a variety of hardened, protective, protruding shapes packed on the surface of a tree similar to scales. As mentioned previously, the bark of some trees qualify as scales or very scale-like. These surface structures are typically implemented as non-overlapping heightmaps such in Wang et al.'s bark model [Wang et al. 2003]. Hart et al.'s early work in bark [Hart and Baker 1996] also utilizes a force flow field which directs bark growth, similar to the concept of water flow directing fish scales.

\section{b. Leaves}

Representing plant leaves is another useful graphics topic. Leaves and flower petals, which are actually modified leaves, are used in a variety of artistic media for decorative 
purposes. Leaves occur on plants in high numbers and are highly visible with flexible flat shapes, making them an interesting challenge to model. The concept of a leaf as a self-similar shape repeating across the surface of a tree ties them closely to the idea of a scale.

Much of the work in leaf modeling focuses on approximating their light reflectance and geometry. As rendering is outside the scope of our work, we will mention geometric methods for leaf generation. There exist a variety of techniques to recreate the shape of leaves including hinged polygons [Bloomenthal 1985], recursive techniques such as Demko et al.'s fractals [Demko et al. 1985] and Prusinkiewicz et al.'s L-systems [Prusinkiewicz et al. 1988], and physically based methods such as Wang et al.'s leaf growth method [Wang et al. 2004]. Some newer work by Runions et al. [Runions et al. 2005] focuses on representing the internal venation structures of leaves, which then directs the shape of the leaf's silhouette.

The variety of methods for representing leaves illustrates the diversity of approaches modeling a surface feature. Although the flat shape of leaves is inappropriate for the thicker shape of scales, they provide insight into using a repeating model based on a template to cover a surface.

\section{Animals}

The significance of animals in Computer Graphics is obvious, as humans appear in the majority of artistic media. Representing other animals, particularly vertebrates, is important as well. Creating a plausible graphical representation of an animal is not an easy task, due to the enormous complexity of biological life. However, there are many useful techniques in CG representations of both human and non-human animal features. There are many methods which focus on the skin and skin-related structures. Because we seek to introduce another skin-related structure for CG modeling, it is 
worth examining these related methods.

a. Skin

Accurately depicting animal skin, especially human skin, is particularly difficult. $\mathrm{Hu}-$ mans have a detailed, instinctive understanding of the appearance of skin, and subtle inaccuracies in artificial skin are detected easily. Because skin is actually a semitransparent flexible tissue matrix, simulating the appearance of skin can be quite complex. Although most skin representations concentrate on rendering skin, some focus on the geometric properties of skin such as Wu et al.'s work on wrinkles [Wu et al. 1997] and Takayuki et al.'s exploration of reptile skin [Itoh et al. 2003].

\section{b. Hair}

Related to the topic of skin is the creation of hair. Most mammalian characters will have some hair partially or fully covering their body. A covering of hair may contain millions of individual hairs, all of which may be long, flexible strands with interesting optical properties. Because of this high number of hairs, individually modeling each hair is prohibitively difficult. A variety of techniques exist to handle the high number of elements in hair simulation. These methods are collected in a recent survey by Ward et al. [Ward et al. 2007].

The problem of hair simulation is related closely to that of scale creation. Both are biological features which grow as large numbers of self-similar elements on the skin of living organisms. In the case of the mammalian pangolin, its scales are actually hairs, blurring the distinction between the two. However, a difference between most CG depictions of hair and scales lies in the hair shape's root or anchor to the skin. Because hairs are filament shapes, and emerge from follicles in the skin, the hair shape itself is not integrated into the underlying surface. The distribution of the hair 
follicles, typically using a random or Poisson distribution, is examined in a study by Streit and Heidrich [Streit and Heidrich 2001].

c. Feathers

Somewhere between scale and hair creation lies feather modeling. Similar to hair and scales, feathers form in great numbers as an epidermal growth on animals. Like scales, feathers form flattened, overlapping shapes. Unlike scales, feathers emerge fully from follicles in the skin much the same way that hair does, and so integration with the skin is less important.

Based upon Dai et al.'s preliminary investigation into feather synthesis [Dai et al. 1995], Chen et al.'s work on creating realistic feathers [Chen et al. 2002] laid major groundwork for feather simulation. Streit's work [Streit 2004], later expanded upon by Bangay [Bangay 2007], explores feather coat morphogenesis. Other work further improves feather modeling, such as Weber et al.'s method of collision avoidance [Weber and Gornowicz 2009]. Beyond that, the body of work remains fairly small. The subject of feathers for CG graphics remains active, however, and many major CG effects companies still explore the topic through proprietary research.

\section{d. The Retina of the Eye}

Some work in representing biological phenomena focuses on more specific topics. Deering's work on synthesizing the human eye's retina[Deering 2005] is one example. The technique simulates the distribution of cones in the human eye through a physically-based growing process where cell positions shift to minimize a repulsion energy.

Eye simulation is particularly notable because it directly ties the concept of Poisson distributions to that of a biological feature. Yellott's work [Yellott 1983] 
with rhesus monkey and human retinas shows that the cells of the retinas form a Poisson-disk distribution. In this distribution, each cell tends to position itself at a similar minimum distance from its neighbor cells. This dense packing approximates blue noise, and creates good visual resolution for the eye.

\section{B. Surface Patterns for Computer Graphics}

To develop a method which creates scales on a surface, we look at CG surface synthesis techniques. There are many ways to depict shapes on the on a polygonal model. The most common approach to representing detail on a surface is by utilizing texture mappings.

\section{Surface Geometry}

Typical methods for generating scale shapes involve extruding the geometry of a surface using displacement maps [Cook 1984], and variations of displacement such as height and tilt textures [Andersen et al. 2009]. Displacements prove useful for adding geometric detail to a surface while retaining manifold topology. However, they are limited because they cannot reproduce high genus surface details, and have trouble reproducing long, thin displacements. Very recent work by Takayama et al. [Takayama et al. 2011] allows mapping of high genus surfaces, but is concerned with mesh cloning of large patches more than synthesis.

Recent work in shell maps [Porumbescu et al. 2005], mesh quilting [Zhou et al. 2006], and volume texturing techniques [Peng et al. 2004] address some of the limitations of displacements and allows complex geometry to be generated in a parameter-

ized space surrounding a mesh surface. However, these techniques are derived from a 2D parameterization of a set of charts covering the mesh. These charts each cover 
large portions of the mesh and create distortion in the scale generation. In contrast, our technique, based on a centroidal Voronoi tessellation, does not require such a parameterization.

\section{Pattern Synthesis}

Methods which alter the geometry of a surface provide the framework for creating surface features, but additional work is needed to guide the surface alteration. For example, a displacement shader may describe how to create a bump on a surface, but does not know where to place the bump. Pattern synthesis methods provide the information which guides this placement of features.

Although there are many different methods for pattern generation, we are primarily interested in creating point-based patterns. In particular, Fleischer et al.'s cellular texture synthesis [Fleischer et al. 1995] provides a biologically-inspired method which grows a pattern of points on a surface. Another concept which proves useful in creating a natural pattern of points on a surface is the centroidal Voronoi tessellation.

Centroidal Voronoi tessellations, or CVTs, generate well-spaced distributions of points [Du et al. 1999] [Du et al. 2002]. They have a variety of applications, including Deering's retina synthesis [Deering 2005] and Streit's feather coat generation [Streit 2004]. CVTs partition a mesh into well-formed regions, as utilized in Cohen-Steiner et al. [Cohen-Steiner et al. 2004], without requiring a 2d parameterization of the surface. Our method derives scale regions, each representing a scale and its surrounding skin, from an anisotropic CVT. This CVT provides positions for scales and defines the shape of each region to be replaced with scale geometry. Further work involving capacity-constrained CVTs [Balzer et al. 2009] constrains the size of Voronoi cells, and Lp CVTs [Lévy and Liu 2010] provide a method for creating stable quadrilateral Voronoi regions. Although CVTs are very expensive to compute, there are ongoing 
efforts to improve CVT computation such as Liu et al.'s recent optimization work [Liu et al. 2009].

While a CVT provides us with positions for scales, we also need scale orientations. Vector fields are a useful tool for defining orientations on a surface, and can guide 2D texture synthesis as shown in [Ying et al. 2001; Fisher et al. 2007; Xu et al. 2009]. These fields also aid in generating 3D textures [Bhat et al. 2004], in geometry orientation as shown by Zhou et al [Zhou et al. 2006], and texture orientation such as shown by Praun et al [Praun et al. 2000]. Additional work in this area includes improved control over vector fields [Zhang et al. 2006] and describing rotational symmetries on surfaces [Palacios and Zhang 2007; Ray et al. 2008]. Our work uses a surface vector field to determine orientation of scales, as well as for anisotropy in the Voronoi tessellation. 


\section{CHAPTER IV}

\section{METHODOLOGY}

\section{A. Scales: Form and Function}

Scales originated as a natural consequence of biological evolution. Many animals and some plants grow them as a form of external protection. These biological scales come in many shapes and sizes. The arrangement of scales on an animal's body, called its squamation, varies from species to species. Even the chemical composition of scales varies between different hardened materials such as a bone, ganoin, or keratin.

A common feature of scales is that they grow from the outer layers of tissue of an organism. In animals the scales grow from the dermis or epidermis. This embedding forms a connective network of scales, functioning as a flexible exoskeleton. Each scale can be viewed both as a distinct element and as part of the connected surface of the scale skeleton.

The connected, embedded nature of scales sets them apart from other protective, symmetric external animal features such as hair and feathers. While hair or feathers are distinct from the skin, scales become part of the skin. In some animals, scales may partially emerge or remain fully submerged under the skin, where instead of directly observing the scales we only see the surrounding skin. As a consequence, representations of scales should consider not only the shape and positions of scales, but their connectivity with the skin or surface of an object.

Most types of scales are oriented in a certain direction. The reasons for this directionality are many. Fish benefit from improved laminar flow from scales directing the flow of water around their bodies, and both the shape and orientation vary depending on the flow of water, habits of the fish, and other environmental factors. 
Scale orientations also influence flexibility. While the segmented nature of scales provides more flexibility than rigid armor, certain movements are restricted based on orientation of the scale. For example, highly concave deformations may be restricted due to scales being flattened against each other or bunched together, creating restricting layers.

\section{Squamation}
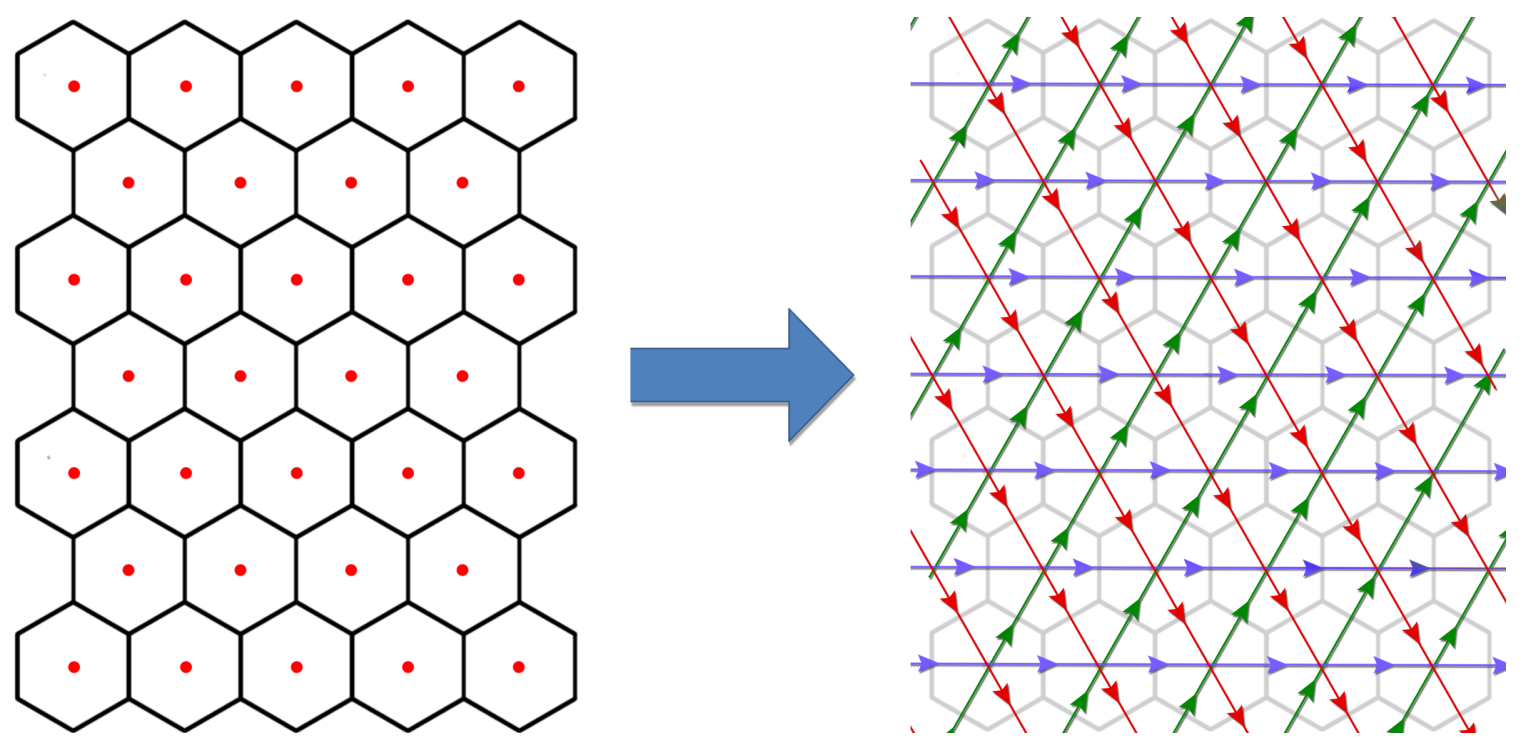

Fig. 9. Illustration of hexagonal tiling, and the three principal directions of its dual. Kardong's work[Kardong 1998] notes these directions appear on many fish.

Scales form both ordered arrangements and disorganized patterns on organisms. These patterns, called squamations, are unique to each species.

\section{a. Ordered Squamations}

Squamations tend to organize into alternating rows, especially on fish. This row alternation forms hexagonal tessellations across the animal. This patterning is a form 
of packing; as scales are pushed against each other under the skin as the animal matures, scales will typically organize into hexagonal arrangements. Kenneth Kardong makes note of this pattern, mentioning that fish scales tend to follow three principal directions[Kardong 1998]. Figure 9 illustrates the relationship between a hexagonal tiling and these three directions.

\section{b. Disordered Squamations}
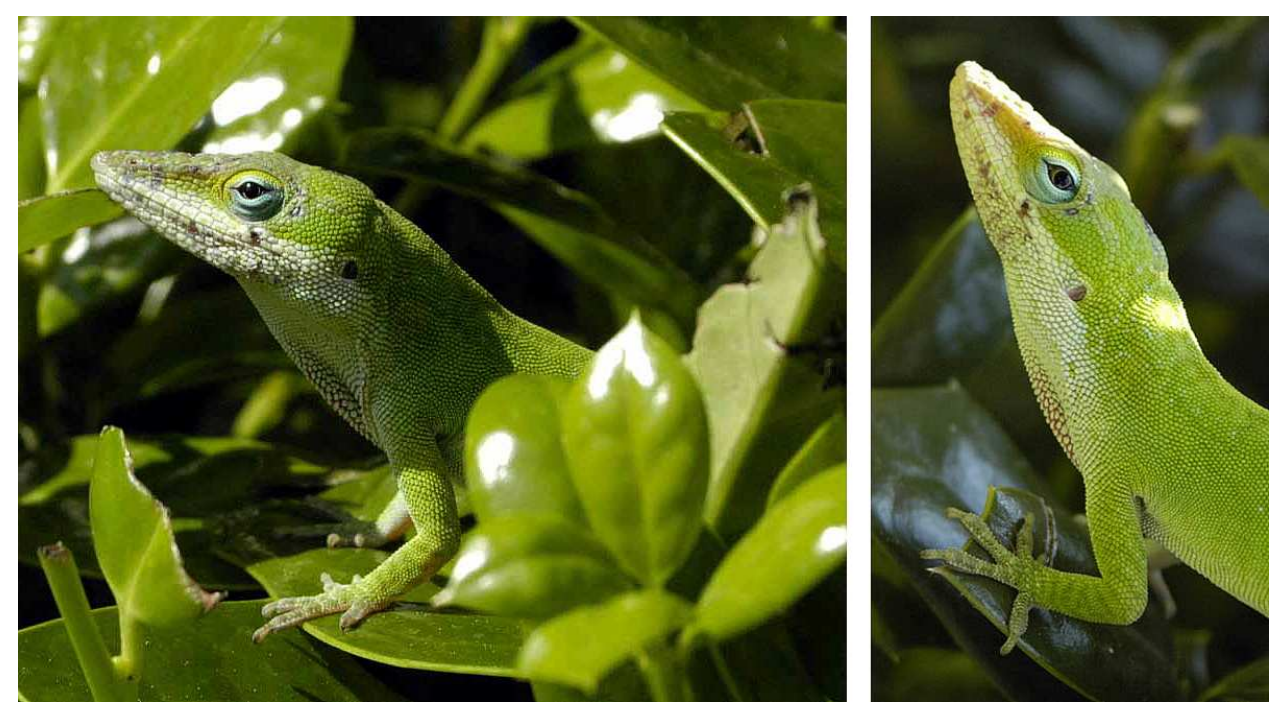

Fig. 10. Example of disordered squamation. The granular scales of anole lizards grow in a pattern resembling a blue noise distribution.

Squamations are not always orderly. Some fish and reptiles such as the anole lizard in Figure 10 exhibit tessellations of scales that appear to be randomly distributed on the surface, instead of forming distinct rows like the ordered squamations. The disordered distribution is not completely random as each scale occupies space and pushes against the other scales, creating a distribution similar to blue noise. If we consider scales to be roughly ellipsoid shapes, their cross-sections as they emerge from the skin are ellipses. The scales are pushed close to each other, with some tissue in 
between, in a kind of elliptical packing. This type of Poisson distribution occurs often in biology, such as in the cells of animal eyes[Deering 2005].

\section{c. Squamation Synthesis}

Creating a natural squamation is the first step in our technique. We will present a plausible arrangement of scales on the surface of a mesh with good spacing. This squamation will position scales in a distribution tending towards hexagonal patterns.
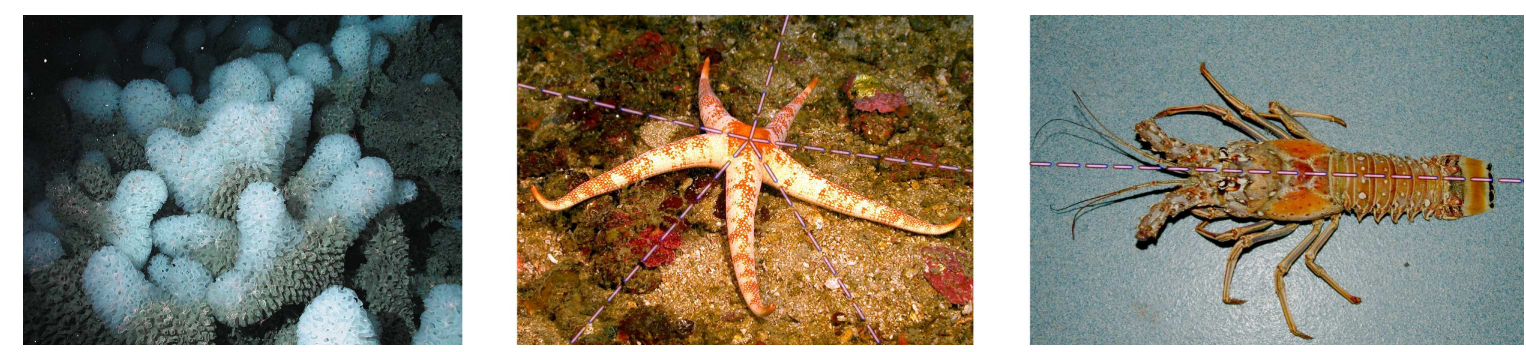

Fig. 11. Left - a white ruffle sponge, an amorphous animal exhibiting very little symmetry. Middle - a starfish, showing radial symmetry. Right - a Caribbean spiny lobster, exhibiting bilateral symmetry. Also note the repetitive symmetry of its 6 legs and tail plates. Photo credits: NOAA Photo Library [NOAA 2011].

\section{Symmetry}

Symmetry plays a significant role in the growth of multicellular animals. The only animals with primarily asymmetric forms are phylum Porifera, the sponges. For other animals, symmetry plays a significant role in their development and locomotion.

\section{a. Biological Symmetry}

Many primitive forms of sea life exhibit radial symmetry. Echinoderms, such as starfish and sea cucumbers, form radially symmetric arms; the bodies of starfish such as seen in Figure 11 are so symmetric that an entire animal can regenerate from 
a single arm. Cnidarians, including jellyfish, coral, and sea anemones, also display a high degree of radial symmetry. Their bodies form highly symmetric dome and cylinder shapes, and have tentacles arranged into rings. Note that although coral colonies typically construct very asymmetric exoskeletons, the polyp itself is highly symmetric.

Plants also exhibit many forms of radial symmetry. The stems and trunks of plants are usually symmetric, as are most flowers.

The form of animal symmetry we are most familiar with is bilateral symmetry. All vertebrates, including humans, are bilaterally symmetric with left and right sides mirroring each other such as the lobster in Figure 11. This symmetry is important for balanced locomotion in vertebrates, and redundancy for surviving injury such as loss of an eye.

\section{b. Repetitive Parts}

A more complex symmetry evident in organisms is their repetitive parts. Repetitive symmetry creates similar features on an animal or plant. Rather than the mirrored duplication of features due to bilateral or radial symmetry, body parts with repetitive symmetry appear to be translated copies of each other.

An obvious example for repetitive symmetry on plants is leaves. The leaves on a plant are morphologically similar, and play an important role in identification of species. Leaves are so self-similar that a plant can be identified using one single leaf.

Scales fit into this category of symmetry due to their self-similarity. Each scale on an animal has similar features, even if the size and shape varies slightly. Like plants and their leaves, species of animals can often be identified by a single scale.

We utilize this repetitive symmetry in our technique to create multiple scales from a single template. Instead of having to create each scale individually, we duplicate 
and slightly modify a single artist-created model to cover a surface. In this manner the artist can concentrate effort into creating a single high quality scale.

\section{Imbrication}
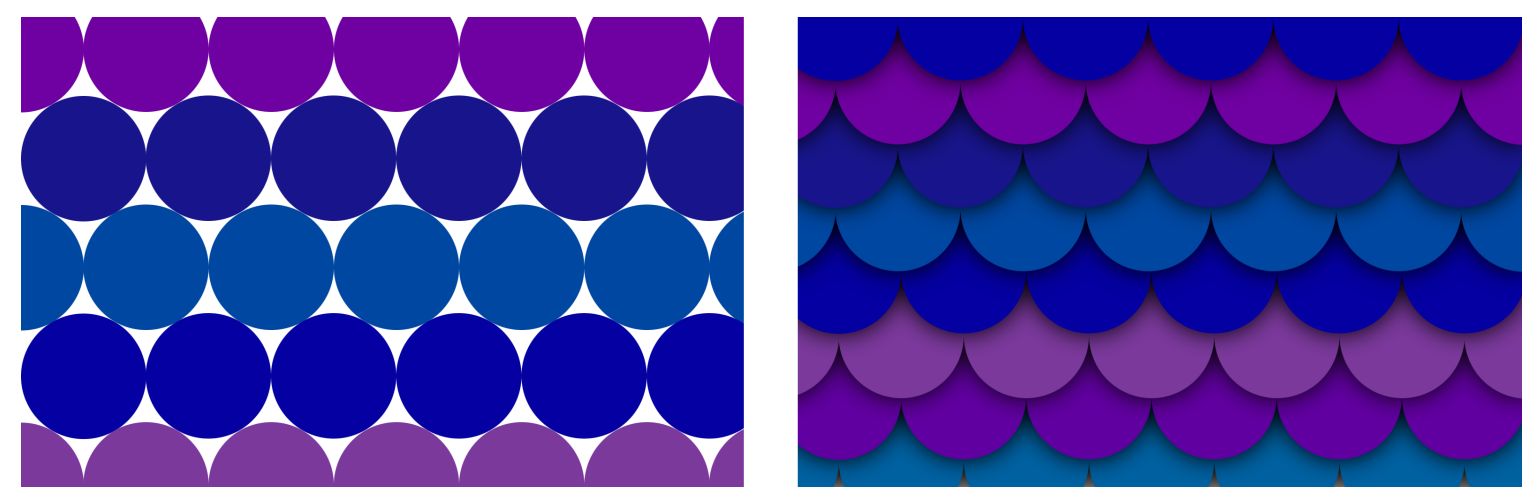

Fig. 12. Left - an optimal circle packing, without overlap. Notice the unavoidable gaps between circles. Right - Imbrication of circles removes gaps, providing full coverage of the surface.

Imbrication, or the overlapping of parts as seen in Figure 12, describes one problem with creation of many animal scales. As scales begin growing deep in the skin of an animal, they grow larger and larger until they overlap each other. This overlapping causes scales to tilt and grow at an upward angle, contributing to their eventual emergence from the skin. The scales tilt in the same direction, typically emerging upwards pointing towards the posterior. Not all animal scales imbricate, either from not emerging fully from the skin or not growing large enough to overlap.

Non-imbricating scales have a disadvantage in flexibility because they cannot move or slide without exposing weaknesses. Because of the lack of overlap, nonimbricating scales often deform together in a mosaic or fuse together. In many cases, the fusing of scales forms scutes or plates, as seen on the placoderms in Chapter II. These fused structures vary wildly from animal to animal, and tend to shape specifi- 
cally to each species's body to form inflexible carapaces.

Imbrication provides significant motivation for our work. Typical techniques for creating scales use displacement maps to generate shapes. These mappings do not allow overlap of shapes, causing an undesirable merging effect on the surface. Although height-and-tilt textures[Andersen et al. 2009] allow overlap, it is unclear if they will work for highly anisotropic scale shapes, especially thin plates. Our method allows for unrestricted overlap by smoothly connecting the bases of scales together. The direction of overlap depends upon the orientation of the scale.

\section{Orientation}

Scales are anisotropic by nature, and their orientation plays an important role. The orientation of fish scales significantly affects the laminar flow of water around the animal. To reduce friction with water, fish scales point towards the posterior in the direction of water flow. In fast-swimming species, and those living in fast-flowing rivers, the shape of the scale is also flattened and smoothed to assist locomotion.

The orientation of fish scales conforms to the lateral line, a feature common to most bony fishes. The lateral line, which appears as a curved line along the flank of a fish, serves as a sensory organ. The lateral line is merely an external feature of a complex system of subdermal sensing organs. The lateral line corresponds to a row of scales on the fish, which are oriented tangentially to the line. Lateral line scales differ from other scales, having bony tubercles that connect them to the lateral system that appear as bumps or small holes. As the animal grows, lateral line scales tend to grow and emerge first, with adjacent scales appearing later in a radiating fashion.

As with other aspects of living creatures, there is a great deal of variety in lateral lines. In addition to being unique to fish, lateral lines appear in a variety of shapes. Some lateral lines appear on a fish's dorsal side, some on the ventral side. Some 
species of fish have several lateral lines, and a few such as herrings do not have an outwardly visible lateral line. The vast majority of fish, however, have a single lateral line and begin scale growth along the line near the caudal fin.

Reptiles and other scaly animals do not exhibit lateral lines. The bodies of these more advanced vertebrates have correspondingly more complex scale patterns, and describing them can be ponderous. The closest analog to a lateral line on a reptile might be backbone scales. Many species of reptile have a row of scales corresponding directly to the spine, or multiple rows of scales along the back of the animal.

The necessity of orientations for scales motivates our technique's directional field and scale spawning algorithm. We utilize the concept of a lateral line to give an artist control over this directional field as well as the overall squamation. The artist draws a lateral line on a surface which constrains the directional field, and guides the growth of new scales on the surface. We represent a set of scales on the lateral line in order to constrain the squamation. 


\section{CHAPTER V}

\section{SCALE CREATION THROUGH SURFACE REPLACEMENT}

To create scales on a model, we divide the problem into two steps. The first step concerns itself with global properties of the scales. These properties include the segmentation of a surface into a squamation which we will position and build the scales from and a directional field which determines orientation of each scale.

Our second step synthesizes each scale. We perform a piecewise replacement of the mesh's surface until it is covered in scales. Each scale's position, orientation, and connectivity information is inherited from the first global step of our method.

\section{A. Squamation}

The pattern of scales on the skin of an animal is called squamation [Sire and Arnulf 1990]. Not only do shapes of individual scales vary from animal to animal, but squamations vary as well. Due to this variety, we wish to develop a general purpose technique for creating a plausible squamation on a mesh's surface. Using the squamation, our method will grow one scale from each patch on the surface.

In Chapter IV, we observe that natural squamations tend towards hexagonal arrangements. These packed hexagonal patterns are reminiscent of Voronoi diagrams of well-distributed points. Specifically, these patterns are similar to those produced by CVTs [Du et al. 1999] where the points in the distribution lie at the centroid of their Voronoi region. These tessellations tend to create hexagonal structures and have

*Reprinted with permission from "Scales and Scale-like Structures" by Eric Landreneau and Scott Schaefer, 2010, Computer Graphics Forum, 29, 1653-1660, Copyright 2010 by Eric Landreneau. 
been used to describe reptile skin[Itoh et al. 2003], feather coats [Streit 2004] as well as other natural phenomena. Because they also do not require a 2D parameterization, as discussed in Chapter III, CVTs are ideal candidates for modeling the squamation.

We represent scales as points on the surface. These points correspond to Voronoi sites, each located at the centroid of a corresponding Voronoi region. In this way, each scale corresponds its own small portion of the surface.

For each scale, we also associate with that scale a direction vector $\overrightarrow{o_{x}}$, which is defined to be in the tangent plane of the surface. We then define a orthonormal coordinate frame at the scale by setting $\overrightarrow{o_{z}}$ to be the normal of the surface and $\overrightarrow{o_{y}}=\overrightarrow{o_{z}} \times \overrightarrow{o_{x}}$. This frame will orient each scale on the surface.

We define each scale's position and direction as its scale-site. The Voronoi diagram of the scale-sites restricted to the surface creates a Voronoi cell associated with each site. To approximate this Voronoi tessellation on the surface, we utilize a method similar to Teichmann et al.'s [Teichmann and Teller Teichmann and Teller].

\section{Voronoi Tessellation Creation}

To create this Voronoi tessellation, we segment a polygonal mesh into Voronoi regions. Each Voronoi region corresponds to a Voronoi site, each of which is identified by a unique, random color for easy user visualization These site colors are first assigned to vertices of the mesh. For each vertex in the mesh, we evaluate a distance metric based on the distance between the vertex and a site. We assign the color of the site with the smallest distance metric to the vertex.

This vertex coloring process gives a simple approximation of the Voronoi tessellation, but has some issues. The precision of the tessellation is dependent upon the original topology of the mesh. Surfaces with more polygons and vertices will approximate the Voronoi regions better, but region edges may still have very jagged edges. 

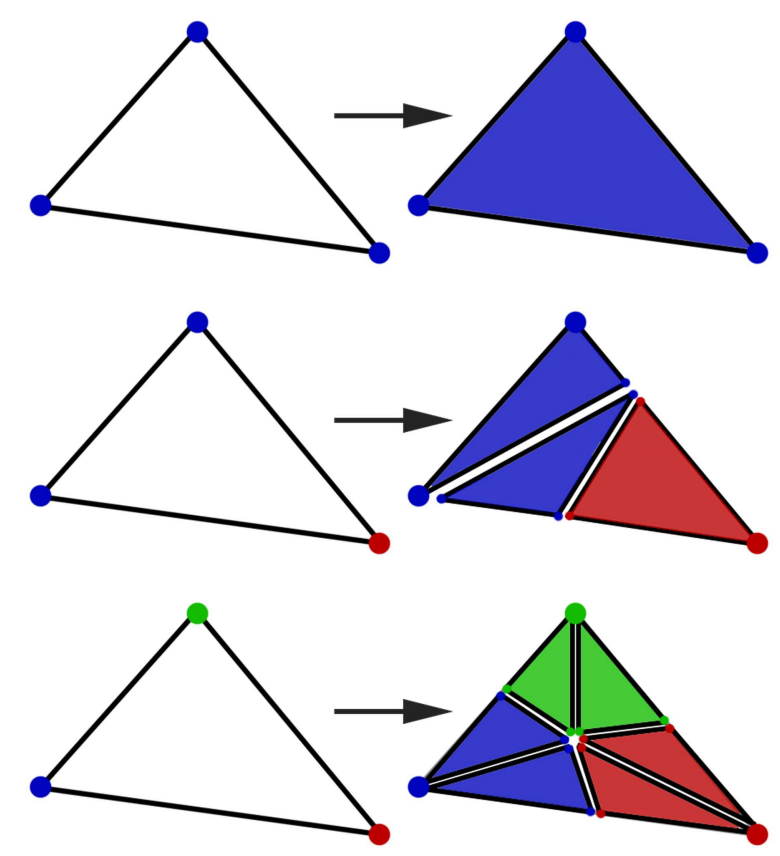

Fig. 13. Faces split and color according to three combinations of vertex colors - one-color (Top), two-color (Middle), and three-color (Bottom).

Because we are segmenting the surface we must color not only vertices but faces, but each face along a boundary has an undefined color due to its vertex colors not matching. To better approximate Voronoi region boundaries, we will subdivide these boundary faces based on observations made by Cohen-Steiner et al.'s work [CohenSteiner et al. 2004].

We observe that triangles of the mesh fall into three classifications - single-color, two-color, and three-color. Single-color faces are faces in which all vertices share the same color. We treat these faces as non-boundary, and assign them associated site-color.

Two-color faces have two vertices sharing a color. The edges shared by different colored vertices split the triangle into three smaller triangles, according to the middle diagram in Figure 13. This split forms three triangles as shown in the figure. This 
splitting creates two new vertices, each on an edge with differently-colored vertices. We use a robust binary splitting operation to find the split point on such an edge which will work with any given distance metric. The reason for using this metric is to allow for altering the distance metric from isotropic to anisotropic, a process we discuss later in this chapter.

We perform the binary split of the two-color edges by evaluating the metric for both Voronoi sites at the midpoint of the line. This splits the line into two segments, one of which is same-colored at endpoints and the other which is differently-colored. We then repeat the process for the segment with differently-colored endpoints in a recursive manner, converging to a medial point which lies along the boundary between the two sites. We found in practice that using four or more recursions produced visually satisfactory results, although the level of recursion is dependent upon the coarseness of the original mesh.

Three-color faces contain three differently-colored vertices. We split this type of face into six triangles in the manner illustrated by the bottom diagram in Figure 13. This process requires creation of three new edge vertices and one interior vertex. We find the edge vertices using the same binary splitting operation as the two-color faces. To find the interior vertex, we must subdivide the surface of the triangle rather than an edge, so we use a recursive face-splitting operation inspired by Loop subdivision [Loop 1987].

The recursive face-splitting operation first breaks a triangle into four smaller triangles by creating edges between midpoints of the triangle's boundary edges. We evaluate the distance metric for all three sites at the centroid of each of these triangles. To choose which of the corresponding triangles to choose for recursion, we make the following observation about the Voronoi boundary. The medial point we are trying to find is the point at which all three Voronoi sites have an identical distance 
metric. It then follows that the difference between each pair of site metrics is also minimized. We sum the differences of these distance metrics to find a combined distance approximation:

$$
\hat{d}(p, s 0, s 1, s 2)=|d(p, s 0)-d(p, s 1)|+|d(p, s 1)-d(p, s 2)|+|d(p, s 0)-d(p, s 2)|
$$

where $d(p, s)$ is the distance metric of site $s$ at point $p$, and $\hat{d}(p, s 0, s 1, s 2)$ is our combined distance metric for three sites. The concern here is not to provide an exact computation, as the exact medial point may lie outside the triangle. We merely desire a point that lies within the triangle boundary, with little difference between distance metrics, and whose calculation can be performed using an altered distance metric, such as the distance to a line. We choose the triangle corresponding to the centroid with the lowest combined distance, and recurse until we find a point.

With faces split and assigned to Voronoi sites, we now have an approximate Voronoi tessellation of the surface. We can find the boundaries of Voronoi regions by merging adjacent same-colored faces, or find the centroid of each Voronoi region by summing the centroids of each triangle contained in that region, weighted by the area of said triangle. Now that we can segment the surface given a set of scale-sites, we need a method that positions and places the sites to produce a good squamation.

\section{Lateral Line}

A simple way to form a squamation is by simply randomly distributing scales (as Voronoi sites) on a surface and use Lloyd's algorithm [Lloyd 1982] to obtain a centroidal Voronoi tessellation. This process generates evenly distributed, mostly hexagonal arrangements of scales, but we desire more control over the squamation. In particular, we wish to provide a mechanism that gives an artist some control over the shape and orientation of the squamation. 


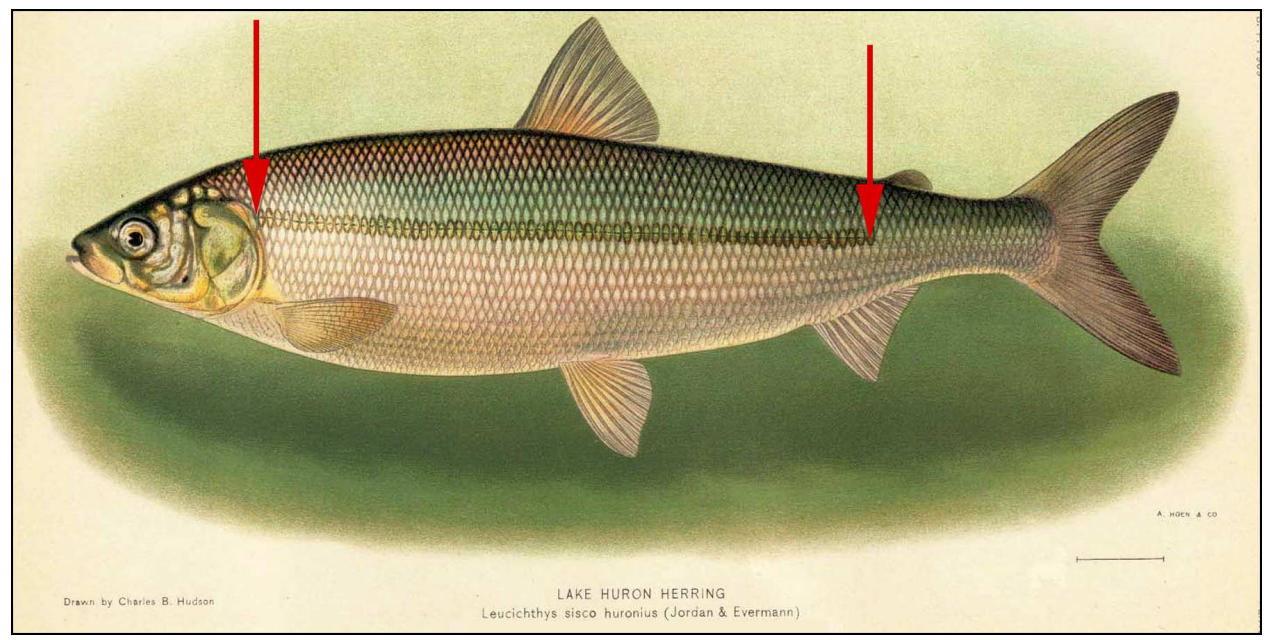

Fig. 14. An illustration of Leucichthys sisco huronius, a Lake Huron herring. Red arrows denote the begin and end of the lateral line, which is highlighted to improve clarity. Image Credit: Charles B. Hudson, NOAA Photo Library [NOAA 2011].

Our inspiration for this control comes from a common anatomical feature of fish called the lateral line. Scale morphogenesis in bony fish often follows the lateral line [Brown 1957]. This lateral line corresponds to a row of scales across the body of a fish, and the squamation will conform to this shape. Figure 14 illustrates a lake herring's lateral line, seen as a faint line across the middle of the body.

Just as a fish's lateral line guides the growth of its scales, we employ an artistdrawn lateral line to guide our squamation. Given a model, the artist draws a single stroke in image space. We project this line from image space onto the model and create scale-sites by sampling this line at intervals of $\gamma$ in model space. This parameter $\gamma$ controls the approximate size of the scales and is user-defined. For each of these scale-sites we also associate an orientation vector $\overrightarrow{o_{x}}$ by sampling the derivative of the lateral line curve, projecting this vector into the tangent plane of the surface and renormalizing.

These lateral line scale-sites serve as seed points for the rest of the scales. We pro- 
vide a simple, coarse seeding algorithm that creates a roughly hexagonal distribution of scales over the surface. This initial distribution will later be refined in subsection 4 . However, the initial distribution of sites affects convergence speed and quality and we have found that this approximately hexagonal seeding pattern produces better results in practice than random seeds.

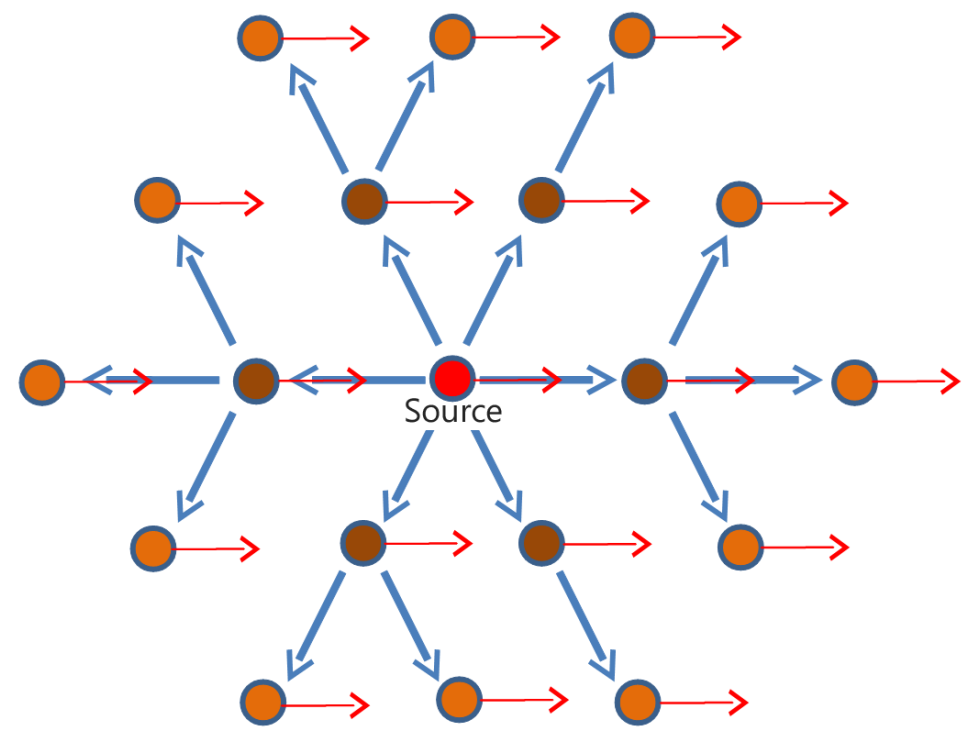

Fig. 15. Scale-sites spawn in 6 principal directions. Arrows in red denote scale-site orientations, and blue arrows represent spawning directions.

Starting with the scale-sites on the lateral line, we perform a hexagonal expansion, shown in Figure 15. For each scale-site, we create new adjacent scales in 6 evenly spaced directions using the vectors spanning the tangent plane $\overrightarrow{o_{x}}, \overrightarrow{o_{y}}$ at distance $\gamma$. For each new scale-site, we project it onto the surface along the normal of its parent scale $\overrightarrow{o_{z}}$. We then create an orthogonal frame for this new scale-site by sampling the normal of the surface at this point $\vec{n}$ and performing a rotation of the parent's frame about the axis $\overrightarrow{o_{z}} \times \vec{n}$ with the angle between $\vec{n}$ and $\overrightarrow{o_{z}}$. If this new scale-site lies outside the region of the mesh scales will be grown on, or the distance from the 


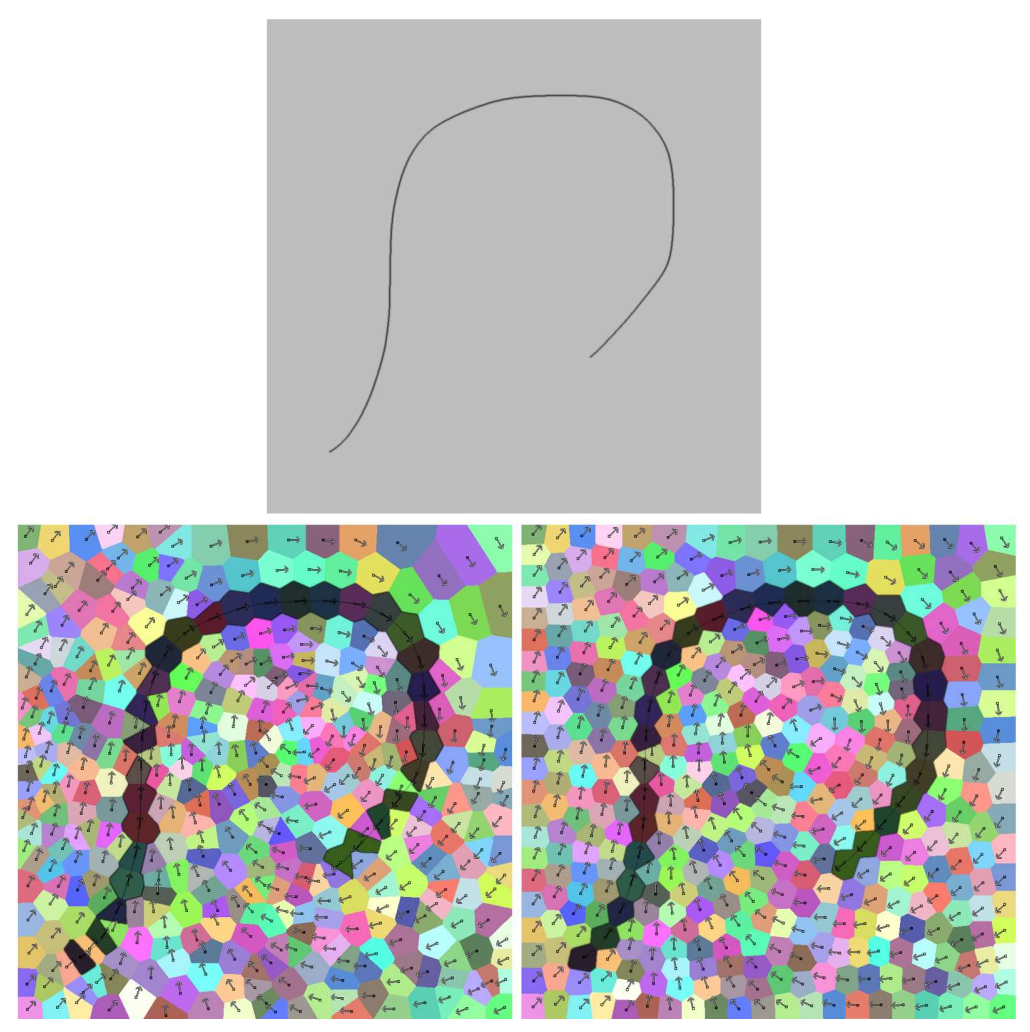

Fig. 16. The user draws a lateral line (Top) to create several scale-sites along that line, colored dark, that we use to recursively spawn new scale-sites to fill the rest of the surface (Lower Left). After running Lloyd's algorithm with the scale-sites along the lateral line fixed in place, the sites converge (Lower Right). The arrows depict the orientation of each site and show how the lateral line influences the vector field.

new scale-site to any existing scale-site is less than $\gamma$, we discard the newly generated scale-site. Otherwise, we create the scale and push a pointer to the new scale onto a queue. This spawning process continues by popping a scale-site from the queue and spawning again, until no more scale-sites can be grown. The resulting configuration of scales covers the mesh with a roughly hexagonal pattern. Figure 16 shows the distribution produced by this recursive spawning process. 


\section{Surface Vector Field}

There may be large deviations between adjacent scale-site orientations depending on spawn order and the shape of the underlying surface. We wish to provide a vector field over the entire surface to control scale orientation such that the orientation changes smoothly over the surface. With this per-vertex orientation field, the orientation of each scale-site will simply be inherited from the closest point on the surface. However, there are several constraints for this vector field. First, we would like to maintain the orientations that the user provided by drawing the lateral line. Second, the orientation field must lie in the tangent plane of the surface.

We create this vector field using a two-level, iterative approach. First, we create a Delaunay triangulation of the scale-sites by taking the dual of the Voronoi diagram. Next, we perform an iterative smoothing process where each iteration consists of three steps. First, we perform a Laplacian smoothing step using the cotan weights [Pinkall et al. 1993] on the direction vectors associated with each scale-site and its one-ring neighbors like so:

$$
\left(\hat{\overrightarrow{o_{x}}}\right)_{i}=\sum_{j}^{n} w_{i j}\left(\overrightarrow{o_{x}}\right)_{j}
$$

where $\hat{\overrightarrow{o_{x}}}$ is the resulting smoothed orientation using cotan weights $w$ for the $n$ neighboring one-ring sites.

To find the cotan weights, we use

$$
w_{i j}=\frac{1}{2}\left(\cot A_{i j}+\cot B_{i j}\right)
$$

where $A_{i} j$ and $B_{i j}$ are the angles opposite to the edge $i j$.

This smoothing process affects the lengths and directions of the vectors so that they are no longer unit and in the tangent plane of the surface. Therefore, we reproject 
those vectors into the tangent plane at each scale-site by finding a new orientations

$$
\begin{array}{r}
\hat{\overrightarrow{o_{y}}}=\vec{n} \times \overrightarrow{o_{x}} \\
\hat{\overrightarrow{o_{x}}}=\hat{\overrightarrow{o_{y}}} \times \vec{n} \\
\hat{\overrightarrow{o_{z}}}=\vec{n}
\end{array}
$$

We also hold the direction vectors for scale-sites along the lateral line constant to act as constraints.

After running this process to convergence, we propagate the direction vectors at the scale-sites to all of the surface vertices within the corresponding Voronoi region and repeat this iterative process again using the triangulation of the surface. Again, we hold the direction vector constant during this process for the nearest surface vertex to each point along the lateral line. This two-level, constrained smoothing process is similar to a multi-grid method and we observe similar increases in speed and stability over simply performing this process on the surface vertices alone. Figure 16 shows the result of this optimization on the direction field.
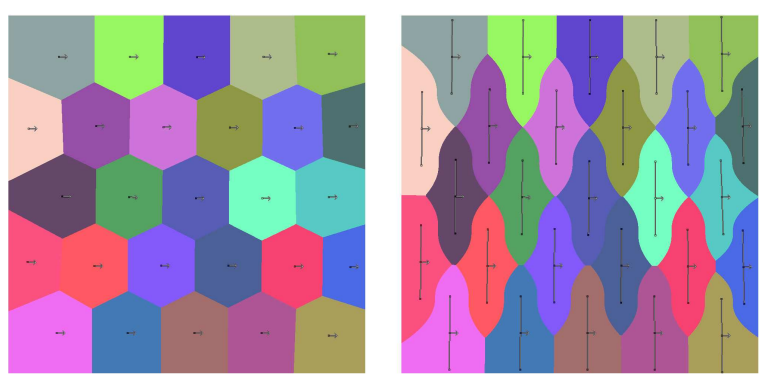

Fig. 17. We represent Voronoi sites as line segments, which can be adjusted to provide an isotropic tessellation (left, $\alpha=0$ ) or anisotropic tessellation (right, $\alpha>0$ ). Arrows denote the orientation of the site. 


\section{Scale Region Improvement}

Once we have a vector field, we create an Centroidal Voronoi Tessellation (CVT) using Lloyd's algorithm [Lloyd 1982] to improve the distribution of the scales. The algorithm consists of a two-stage iterative process. In the first stage we find the centroid of each Voronoi region, then move each scale-site to the centroid of its associated region. The site projects onto the surface by tracing a ray along $\overrightarrow{o_{z}}$, then using the normal at that surface point as the new $\vec{z}$. Once all sites have been moved to their new positions, we create a Voronoi tessellation from the new site configuration. This process of moving sites, then re-tessellating continues until sites eventually converge to the centroids of their regions. While this produces a Poisson distribution of sites constrained to the surface, the shape of the Voronoi regions will be isotropic. However, scales can form anisotropic regions where they intersect the skin, due to being flattened discs which emerge at an angle. To represent these anisotropic regions we must alter the way we create the CVT.
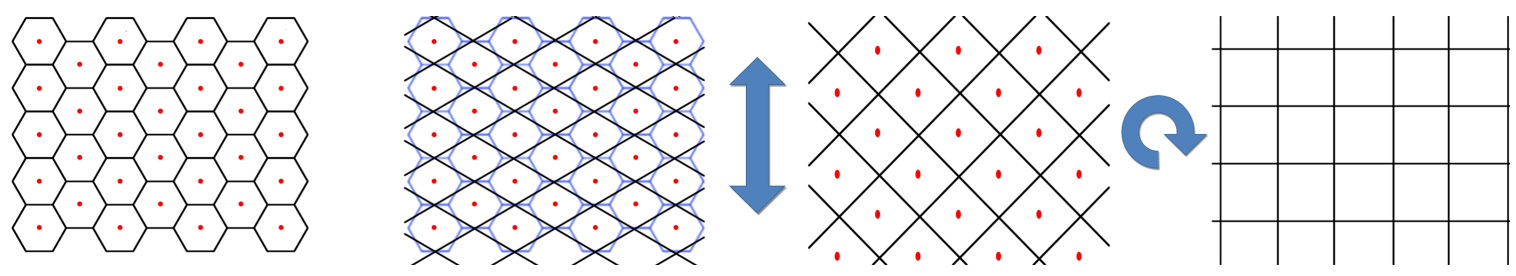

Fig. 18. Illustration of the homotopic relationship between a hexagonal tiling and rhombic tiling's face centers.

To create an CVT with anisotropic regions we keep much of Lloyd's algorithm the same: compute the Voronoi region associated with each scale-site and move the site to the centroid of its Voronoi region. We create anisotropy by modifying the Voronoi region itself to compute the distance to a line segment instead of a point. For each scale-site, we extrude a line from the site's position in the directions of $\overrightarrow{o_{y}}$ 
and $\overrightarrow{o_{y}}$ by a user-given amount $\alpha \geq 0$ that controls the amount of anisotropy as shown in Figure 17. For scale-sites that are not part of the lateral line, we move the sites to the center of their Voronoi regions and reproject them onto the surface. We then update the frame associated with these scale-sites by setting $\overrightarrow{o_{z}}$ to the normal of the surface at that point and sampling $\overrightarrow{O_{x}}$ from the orientation field on the surface we described in subsection 3 .

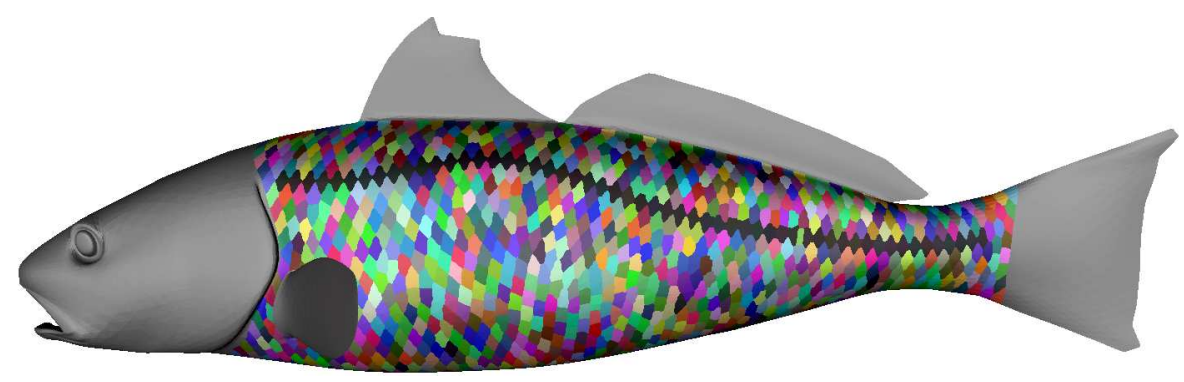

Fig. 19. Voronoi tessellation on a model. We tessellate the surface of a model into Voronoi regions, where each region represents a single scale.

There is a homotopic relationship between rhombic tilings and hexagonal tilings' face centers, as shown in Figure 18. Although we could use an anisotropic tessellation as described by Du et al.[Du and Wang 2005], using a line segment for anisotropy will express this homotopic relationship by distorting hexagons into approximately rhombic regions rather than the elongated hexagons in Du et al.'s work. Many real animal scales such as ganoid fish-scales express rhombic patterns, so this hexagonalto-rhombic alteration is often desirable. These rhombic shapes also interleave more than hexagonal regions, which allows protrusions of the scales to overlap more as seen in Figure 19.

The surface is segmented into distinct regions based on our anisotropic CVT. For each of these regions, we wish to fill that region with geometry representing a single scale. This scale will conform to the shape of the region while connecting seamlessly 

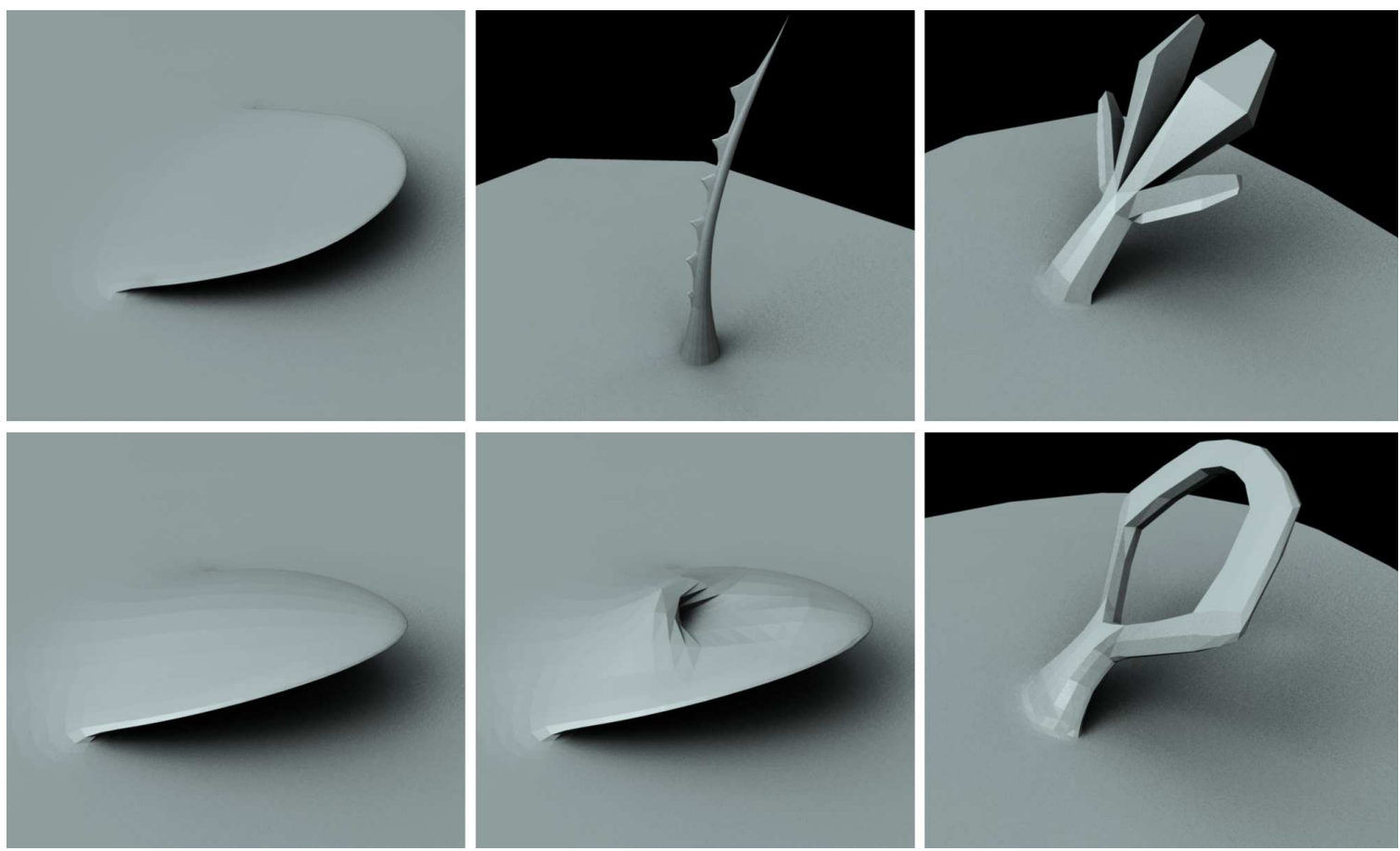

Fig. 20. Examples of different artist-created proxy models.

to neighboring scales.

\section{B. Scale Synthesis}

Since scales tend to be self-similar, we require the artist to provide only a single model of a scale, which we call the proxy model. Examples of these models are shown in Figure 20. For ease of use, we request that the scale protrude from the $x y$ plane and be oriented such that the $\mathrm{y}$-axis contains the greater amount of anisotropy since we will orient the $\mathrm{y}$-axis in scale space with the vector $\overrightarrow{o_{y}}$ for each scale site. Given an anisotropic CVT, we replace each region corresponding to a scale with the proxy model, which we cut and fit to the boundary of that region. While we could use a method like SnapPaste [Sharf et al. 2006] to create these scales, we wish to replace the entire surface with scales that connect to each other instead of the isolated 


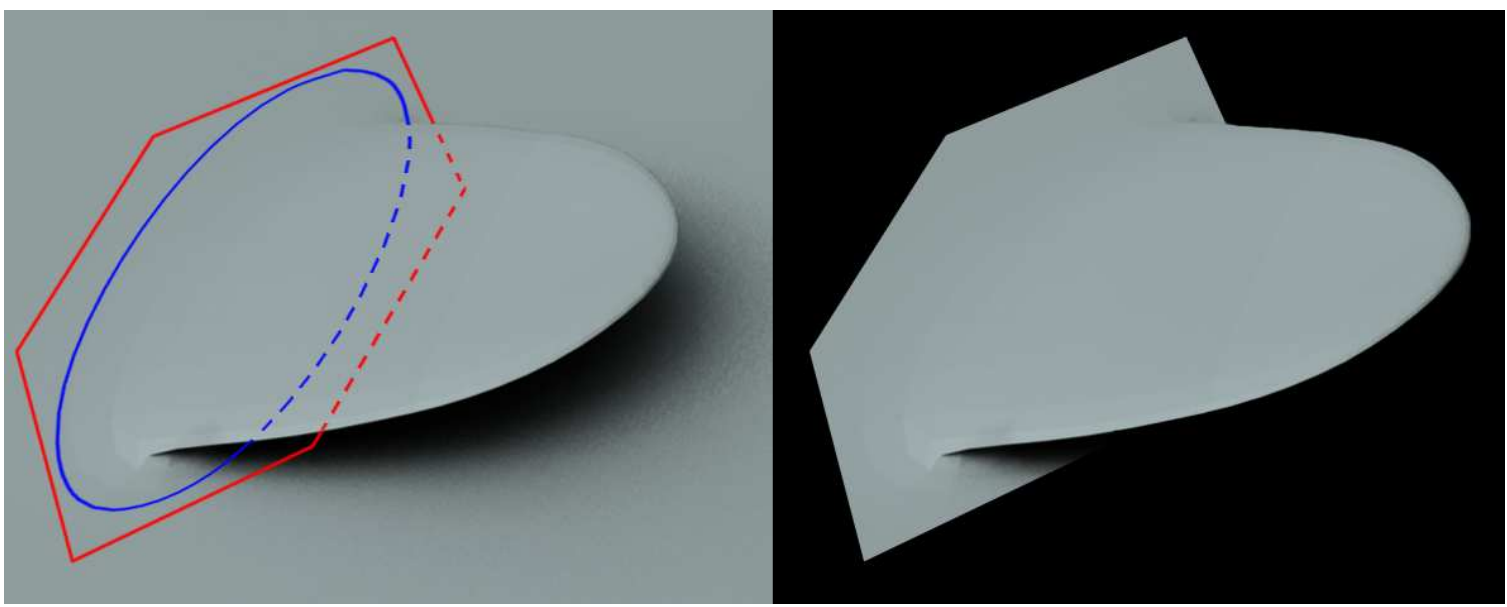

Fig. 21. Scale cutting, before(left) and after(right). A cutting boundary (red) derived from the scale's Voronoi region resizes until it fits the safety region(blue).

replacements of SnapPaste. Consequently, we create an algorithm that allows us to process each scale independent of all other scales on the surface, which lends itself well to a parallel implementation.

Our first step is to transform the region corresponding to a scale from global coordinate space to the proxy model's local coordinate space. We could perform this transformation using some localized parameterization method such as Liu et al. [Liu et al. 2008], but most of these regions tend to be nearly planar and simple projection suffices. Let the scale-site be located at $p$ with an orthonormal frame $\overrightarrow{o_{x}}, \overrightarrow{o_{y}}, \overrightarrow{o_{z}}$. Given a point $v$ part of the scale's Voronoi region, we transform this point using $\hat{v}_{x}=(v-p) \cdot \overrightarrow{o_{x}}, \hat{v}_{y}=(v-p) \cdot \overrightarrow{o_{y}}$. With the Voronoi region's polygon now in the the same space as the proxy model, we can resize the polygon and begin the cut operation. 


\section{Cutting}

To cut the proxy model such that it fits the flattened Voronoi region, we use the boundary of the flattened Voronoi region as a cutting template. We also resize the boundary so that it closely fits the protruding region of the scale. We begin by finding a safety region on the proxy mesh. We mark each face containing a vertex above a user-specified $z$ threshold as unsafe to cut. Next, we find the outer boundary of the proxy mesh as edges adjacent to a single face. A flood-fill marks faces inward from this outer boundary as safe to cut, stopping when it reaches the faces marked as unsafe. Figure 21 illustrates this safety region and cutting boundary.

Once we have marked the region of the proxy model that is unsafe to cut, we iteratively resize the flattened Voronoi region to the scale. First, we fit the Voronoi region to the safety region so that the scale fills as much of the Voronoi region as possible.

To perform resizing, we first translate the flattened Voronoi region by a vector from its centroid to the centroid of the safety region. Next, we uniformly scale the Voronoi region by half repeatedly until it intersects the safety region, then double its scale repeatedly until it does not intersect. The Voronoi region is now at a size $\kappa_{1}$ where it does not intersect, but will intersect the safety boundary if halved in scale to a size $\kappa_{2}$. The closest fit of the region is now bounded by the current range $\left[\kappa_{2}, \kappa_{1}\right]$. We then perform an recursive midpoint resizing, in which we test intersection at $\kappa_{\text {mid }}=0.5\left(\kappa_{1}+\kappa_{2}\right)$, selecting a new range $\left[\kappa_{2}, \kappa_{\text {mid }}\right]$ if intersection fails, $\left[\kappa_{\text {mid }}, \kappa_{1}\right]$ if intersection occurs.

We then find the closest edge of the safety region to the boundary and move the Voronoi region along that safety edge's normal until another safety edge is hit. We label the distance moved as $\rho$. The Voronoi region repositions at the midpoint, at 

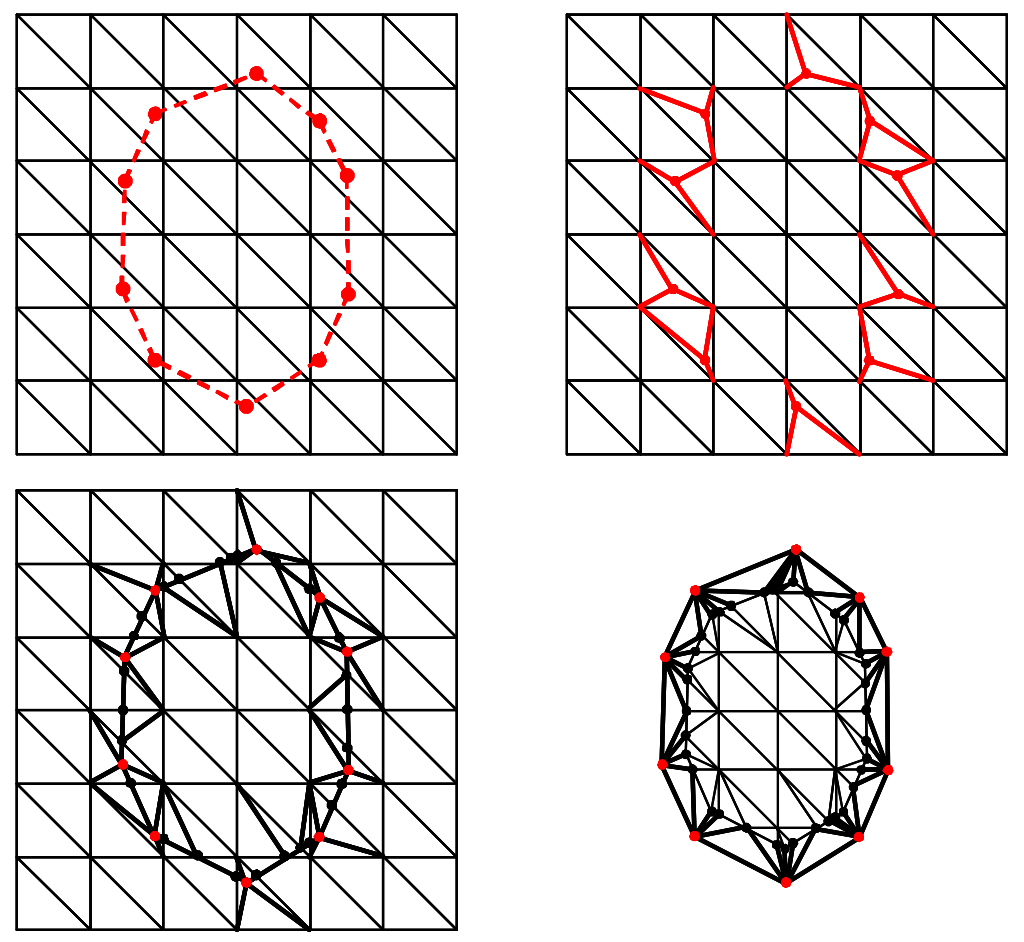

Fig. 22. Top left:triangulation of a proxy model with the boundary of the flattened Voronoi region in red. Top right: stellating the proxy model triangles using the boundary vertices. Bottom left: performing edge splits. Bottom right: shrinking the triangulation and stitching to the boundary.

distance $\frac{\rho}{2}$ from its original position. Repeating this process of scaling and translating the flattened Voronoi region generates a fit that conforms to the shape of the scale.

An affine transformation matrix $T$ records movement information during this process of resizing. For each uniform scale or translation we perform, we correspondingly apply that operation to $T$. This matrix is stored so we can later invert it to find a transformation back to the boundary's original size and position.

Next, we cut the proxy model to fit the flattened Voronoi region using the boundary of the Voronoi region. We trace each boundary vertex vertically along a $z$-axis ray until the ray hits a triangle of the proxy model, which is then stellated by connecting the projected vertex to the three vertices of the triangle (Figure 22 top right). After 
performing this repeated stellation, some of the edges of the boundary may intersect edges of the proxy model's polygons. For these edges, we introduce new vertices where the edge between adjacent boundary vertices intersects the edges of the proxy model (Figure 22, bottom left). After this process is complete, we can discard the polygons of the proxy model that lie outside the flattened Voronoi region.

This process produces a proxy model whose boundary conforms to that of the Voronoi region, but introduces new vertices when boundary edges intersect edges of the proxy model. We would like to create a water-tight result when stitching adjacent scales together and, therefore, cannot introduce new vertices along the boundary. Our solution is to scale the cut proxy model to be slightly smaller. After scaling, we connect the boundary vertices of the Voronoi region to the corresponding vertices of the scaled/cut proxy model and triangulate these quadrilaterals by connecting the remaining proxy model boundary vertices to the closest boundary vertex of the Voronoi region (Figure 22 bottom right). The result is a proxy model whose boundary conforms exactly to the boundary of the original Voronoi region.

\section{Merging}

Using the cut and triangulated model of the scale from subsection 1, we now map this scale onto the Voronoi region on the surface model. First, we move the vertices of the proxy model to match the original flattened Voronoi boundary by an inverse transformation, $\hat{v}=T_{-} 1 v$. Next, the vertices transform from the proxy model's local space to global space using $\hat{v}=p+v_{x} o_{x}+v_{y} o_{y}+v_{z} o_{z}$.

The proxy model protrudes from a flat plane, but the Voronoi region on the surface is typically curved, so the transformed model will not match the Voronoi region on the surface. Therefore, we must deform the cut proxy model to fit onto the surface smoothly. 
We use a Laplacian deformation with implicit optimization [Sorkine et al. 2004] to deform the surface due to its simplicity, though many other deformation methods could be used. The deformation minimizes an energy metric

$$
E\left(V^{\prime}\right)=\sum_{i=1}^{n}\left\|\delta_{i}-\mathcal{L}\left(v_{i}^{\prime}\right)\right\|^{2}+\sum_{i=m}^{n}\left\|v_{i}^{\prime}-p_{i}\right\|^{2}
$$

where $\delta_{i}$ is the Laplacian coordinate of a vertex in the undeformed mesh, $\mathcal{L}\left(v_{i}^{\prime}\right)$ is the Laplacian coordinate of the deformed vertex, $p_{i}$ is the original position of a border vertex, border vertices have indices $m \ldots n$, and $V=v_{1}, \ldots, v_{n}$. We find the Laplacian coordinate of each vertex using the cotan-weighted summation:

$$
\delta_{i}=v_{i}-\sum_{j} w_{i j} v_{j}
$$

with cotan weights $w_{i j}$ for each vertex $j$ in the one-ring.

To make sure that the mesh is water-tight with adjacent scales and that the smoothness of the scales matches that of the surface along the scale boundaries, we constrain the positions of the outer two rings of vertices for the cut proxy model. Notice that the flattened Voronoi region provides a map from the $x y$-plane in scale space back to the corresponding point on the surface. For each point in the 2-ring of the boundary, we find the triangle in the flattened Voronoi region containing the point and map the point to the surface using its barycentric coordinates in that triangle. These points become hard constraints for the local deformation that, along with the Laplacian optimization, create a linear system of equations for the positions of the deformed vertices. 


\section{Summary}

In summary, our proposed method will procedurally generate an even distribution of scales on a surface. An artist controls this scale creation by drawing a lateral line on the surface, and provides a proxy model which replaces geometry at each scale. This process provides a simple, novel interface for creating a complex pattern of scales on a mesh.

Our scale generation technique will produce a watertight, topologically manifold surface. The model will be suitable for simplification and subdivision operations, such that the polygon count of the final model can be easily adjusted by popular mesh optimization techniques such as Garland's QEF simplification[Garland and Heckbert 1997].

Our scale generation technique is general enough to also encompass other skin features, such as porcupine quills or sea urchin spines. As long as a proxy model can be cut by a scale region's boundary polygon, the proxy models can be fit to the surface. This means that long, spindly structures such as spines or feathers can be easily created by our method. 


\section{CHAPTER VI}

\section{RESULTS}
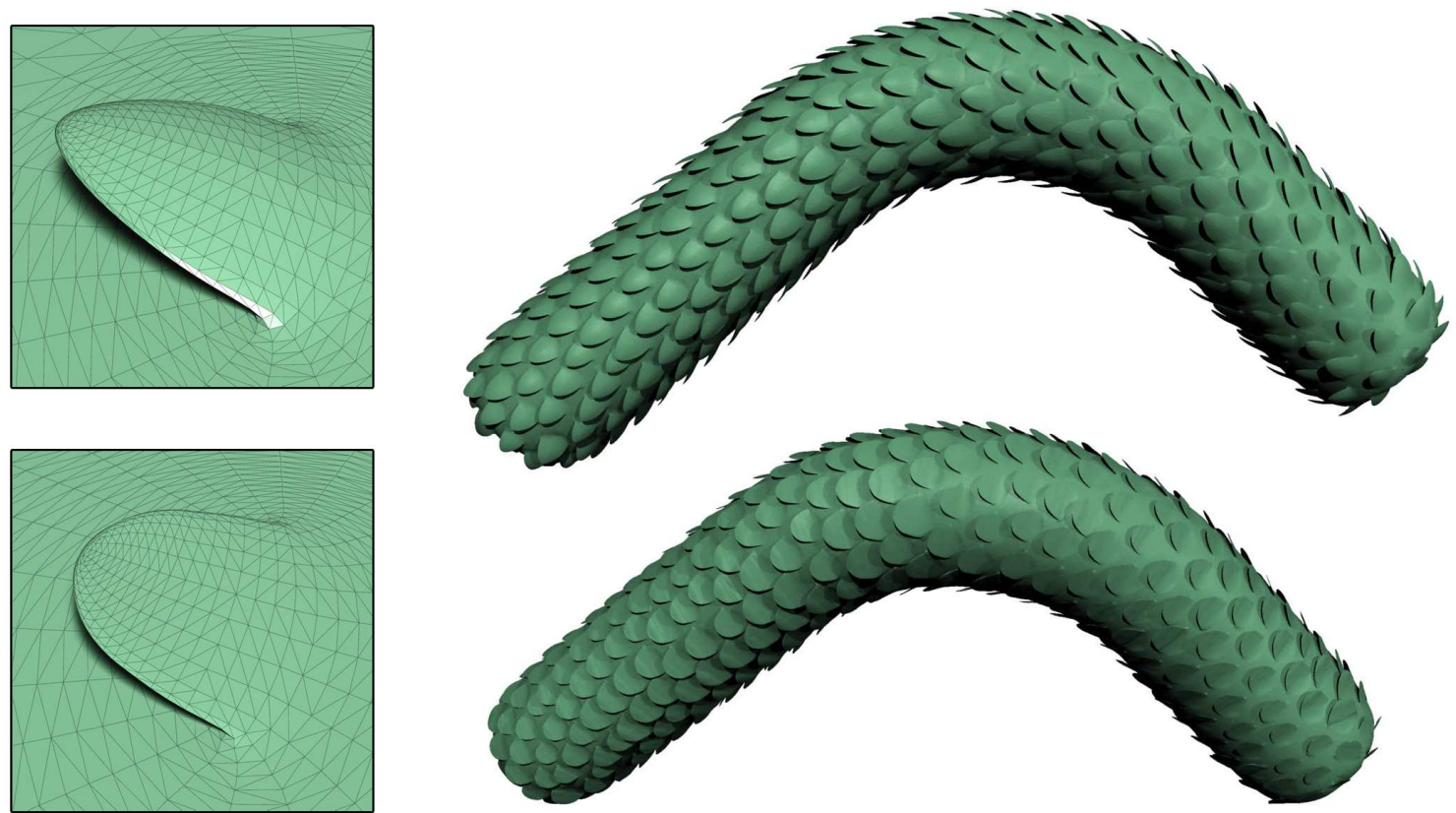

Fig. 23. Above and below right - Bent cylinders with two types of scales applied. Above and below left - The pointed and rounded scales applied to the cylinders.

We tested our method on a variety of meshes, using several different proxy models.

We first tested a bent cylinder, shown in Figure 23. The user drew a small lateral line on the upper right portion of the cylinder, and our algorithm automatically generated scales covering the remainder of the mesh. Note that the direction of the scales smoothly change with the shape of the cylinder, following the surface vector field.

*Reprinted with permission from "Scales and Scale-like Structures" by Eric Landreneau and Scott Schaefer, 2010, Computer Graphics Forum, 29, 1653-1660, Copyright 2010 by Eric Landreneau. 


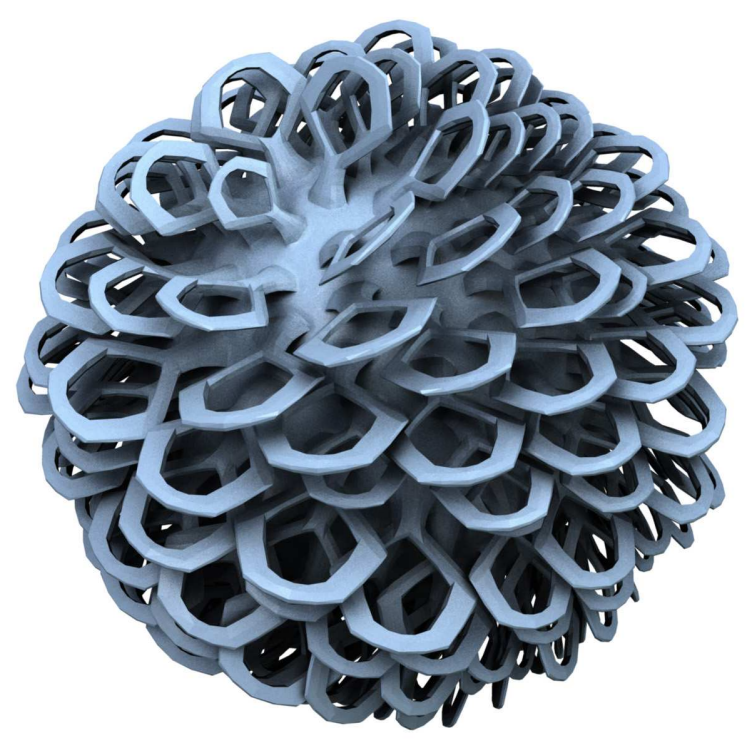

Fig. 24. A sphere covered with high genus scales.

Our second example, in Figure 24 shows the result of testing a high genus proxy model, containing a hole, applied on a sphere. This illustrates an advantage over displacement techniques. Surface displacement alters existing geometry by subdividing the surface and modifying vertex positions but cannot alter the genus of the surface. Displacement works best with simple structures such as bumps or ridges where the surface displaces along the normal. Complex structures such as serrations require specialized displacements like height-and-tilt textures [Andersen et al. 2009]. In contrast, our method performs geometry replacement, which allows us to create complex, high-genus scale structures without any displacement.

We show a practical application of our technique by applying scales to a model of a redfish, shown in Figure 25. A user painted a region of the model as a scale growth region to limit the region we allow scales to grow in. The user then drew a lateral line across the body to produce the squamation present in the image. Our method creates a plausible distribution and orientation of the scales using this input 

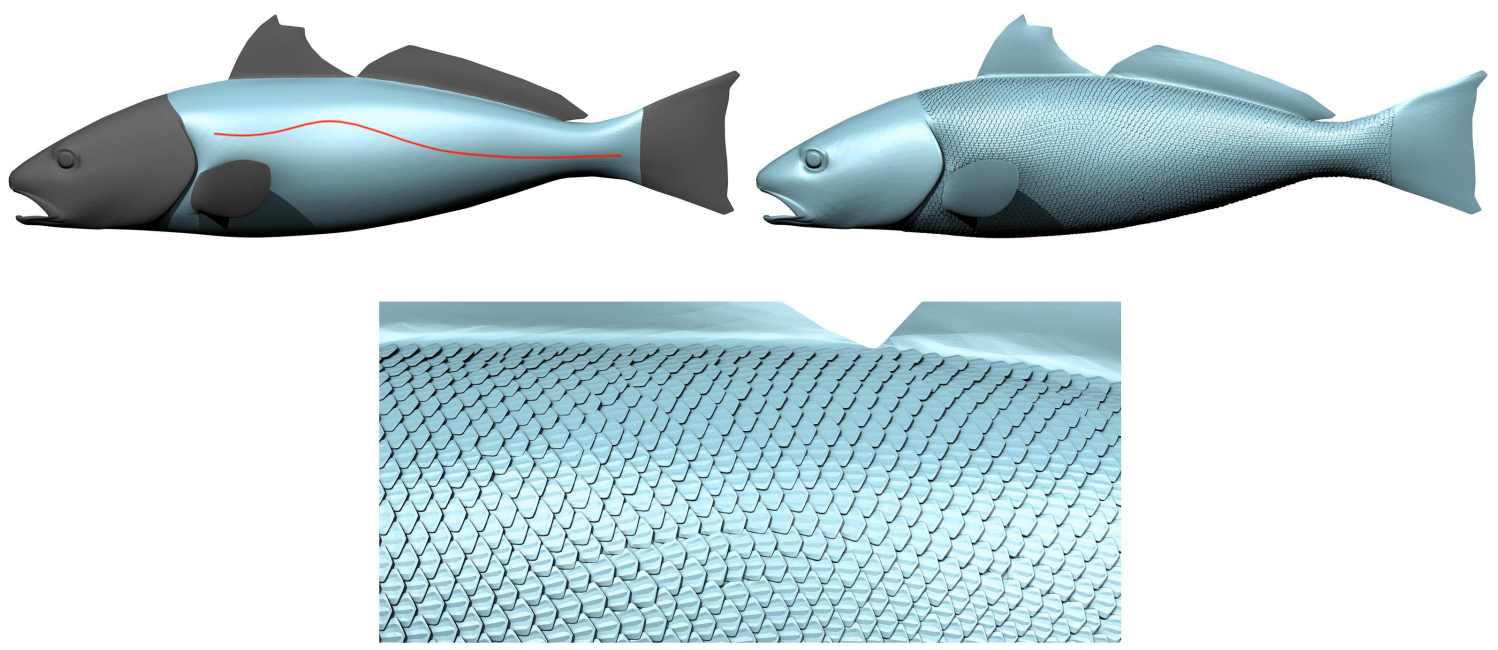

Fig. 25. A fish covered with 3306 scales using our technique. The scale generation region is blue-colored (top left). The user controls the distribution and orientation of the scales by drawing a lateral line on the fish. Our technique uses these inputs to generate a water-tight model covered with scales (top right). The closeup (bottom middle) illustrates the detail achieved by our method.
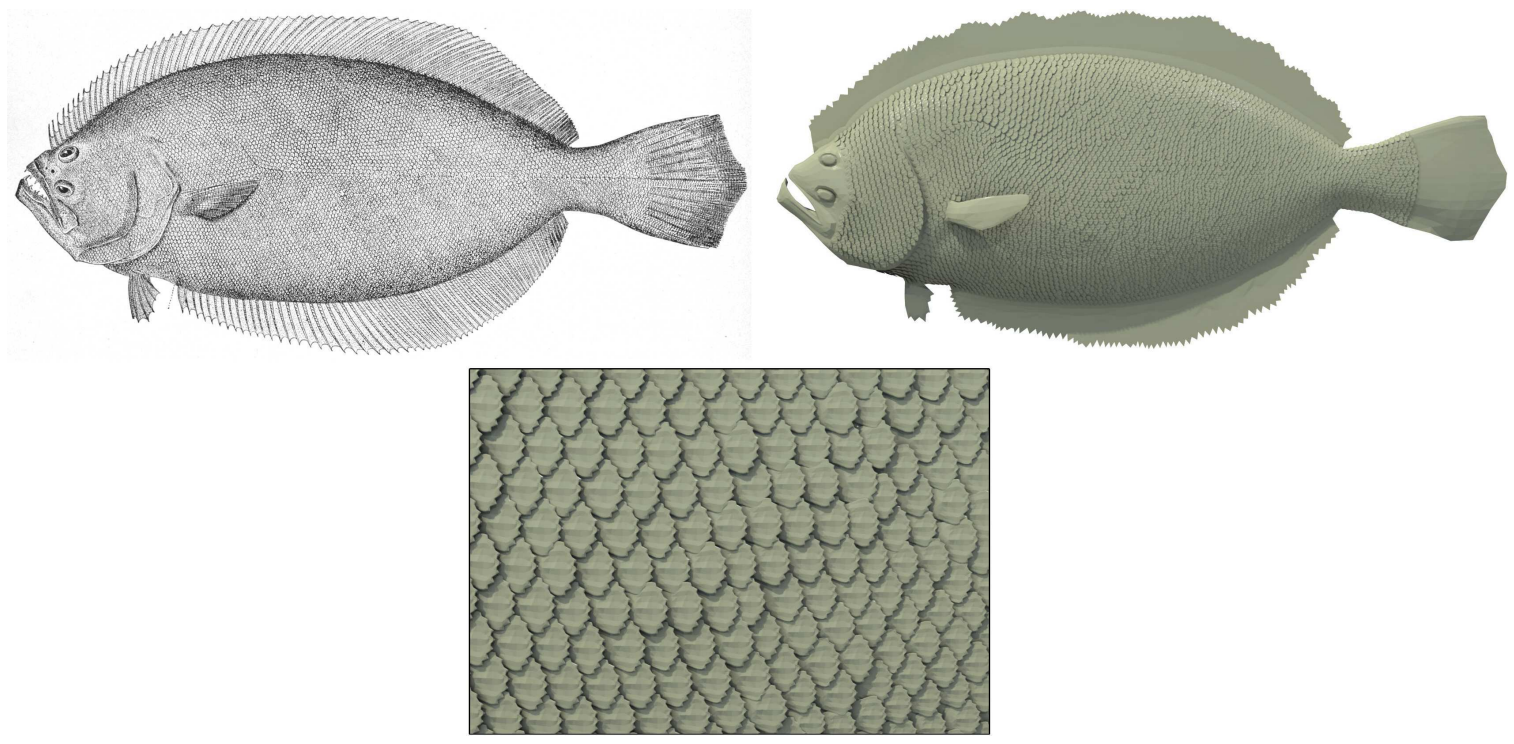

Fig. 26. Left - an illustration of a flounder (drawing credit: H.L. Todd, NOAA Photo Library [NOAA 2011]) Right - A flounder with ctenoid scales applied. Bottom - a closeup of the flounder's scales. 
and produces a water-tight surface. We test a second fish, the flounder, in Figure 26 and apply ctenoid scales to its body.
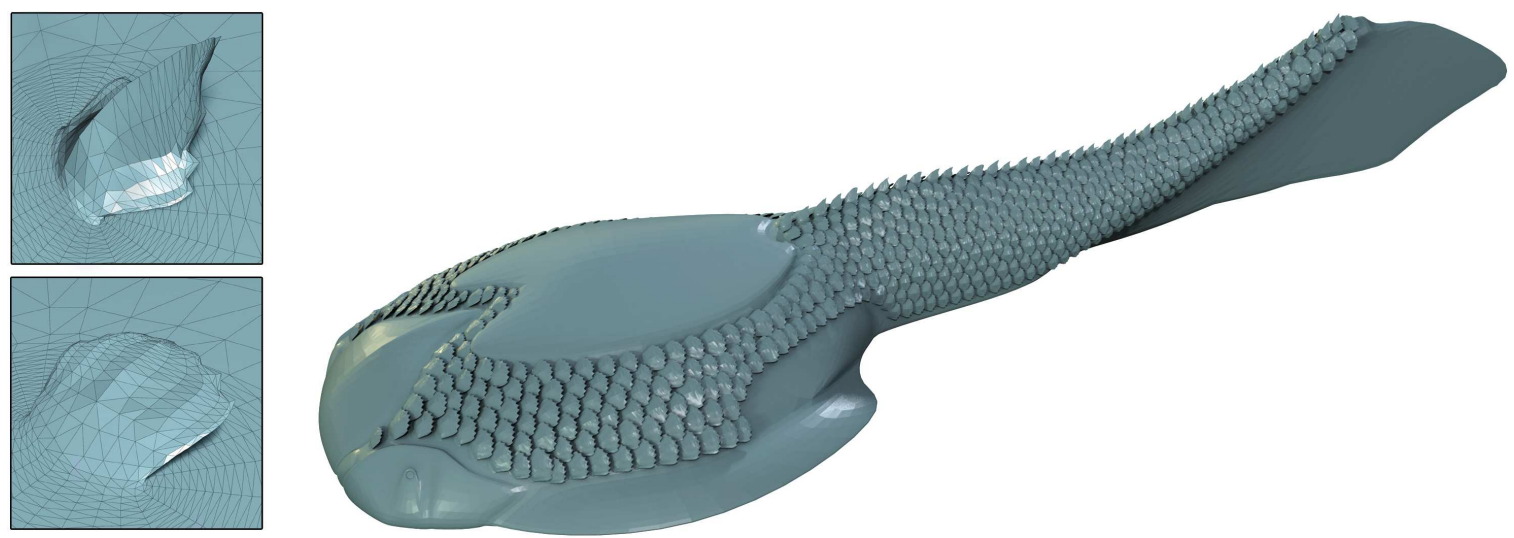

Fig. 27. Scales applied to the extinct ostracoderm fish Drepanaspis. Two proxy models were used, for the backbone along a lateral line (upper left) and for the rest of the body (lower left).

We find that our technique is particularly useful for modeling fish that no longer exist. The primitive ostracoderm Drepanaspis exists only in ancient fossils. These fossils provide poor reference for artists, and there is a great deal of speculation as to how the animal really looked. In Figure 27, we produce a plausible scale-covered surface for this extinct animal.

In another test using two different scales, we create scales on the body of a lizard, in Figure 28. The lateral line's scales use a long, smooth spine for their proxy models whereas other scales use a shorter, serrated spine. For such long, spindly structures, standard displacement mapping would produce heavily distorted triangulations due to the difference in surface area. Furthermore, the serrated pattern is incompatible with standard displacement mapping because a single vertical displacement would map to several points on the spine.

We applied a block-shaped scale to the head of an anole lizard in Figure 29. 

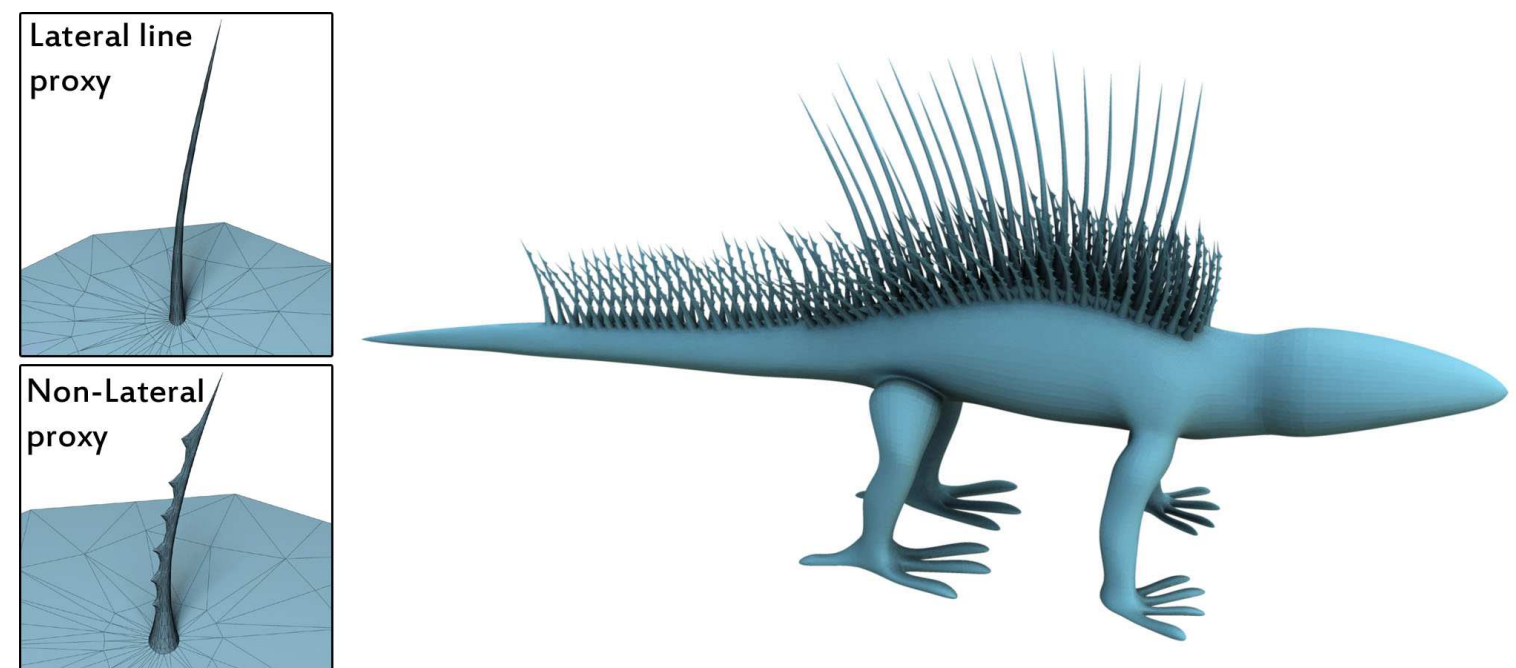

Fig. 28. A lizard model with a lateral line painted down its back. Scale sites on the lateral line use a smooth spine for the proxy model(upper left), and the other sites use a barbed spine (lower left). The resulting surface smoothly joins both types of scales together.
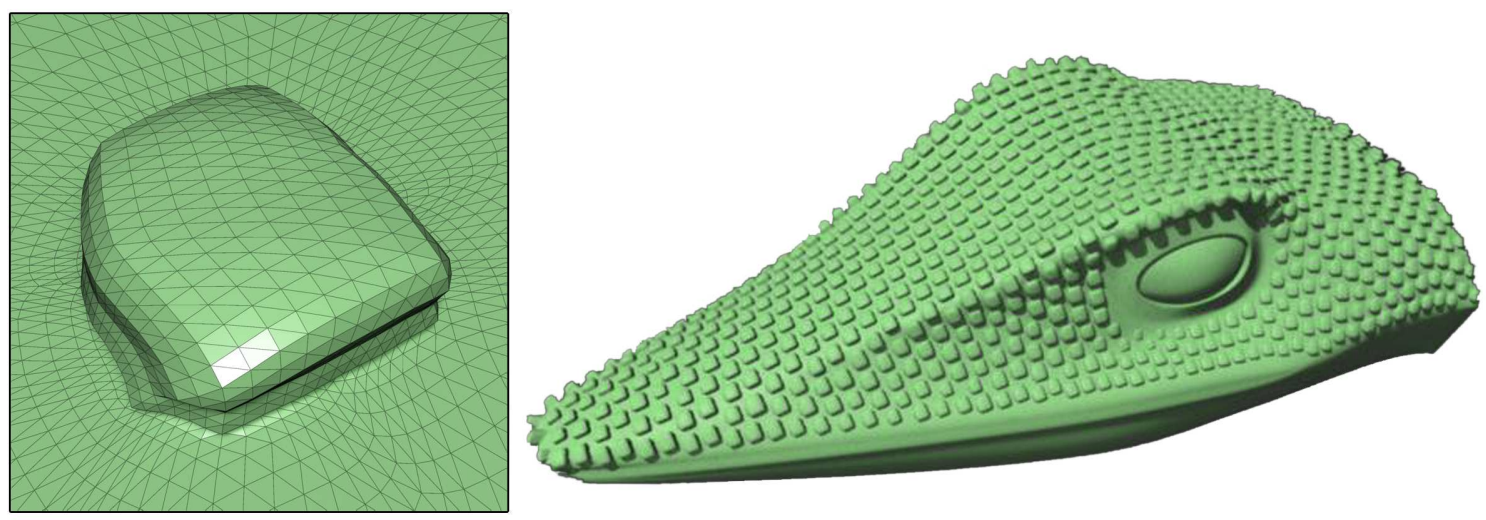

Fig. 29. Lizard head with block-shaped scale (left) applied. 
These scales are motivated by the granular, non-imbricating scales of many reptiles. Even with holes in the growth region for the eyes, the distribution of scales remains even and seamless.
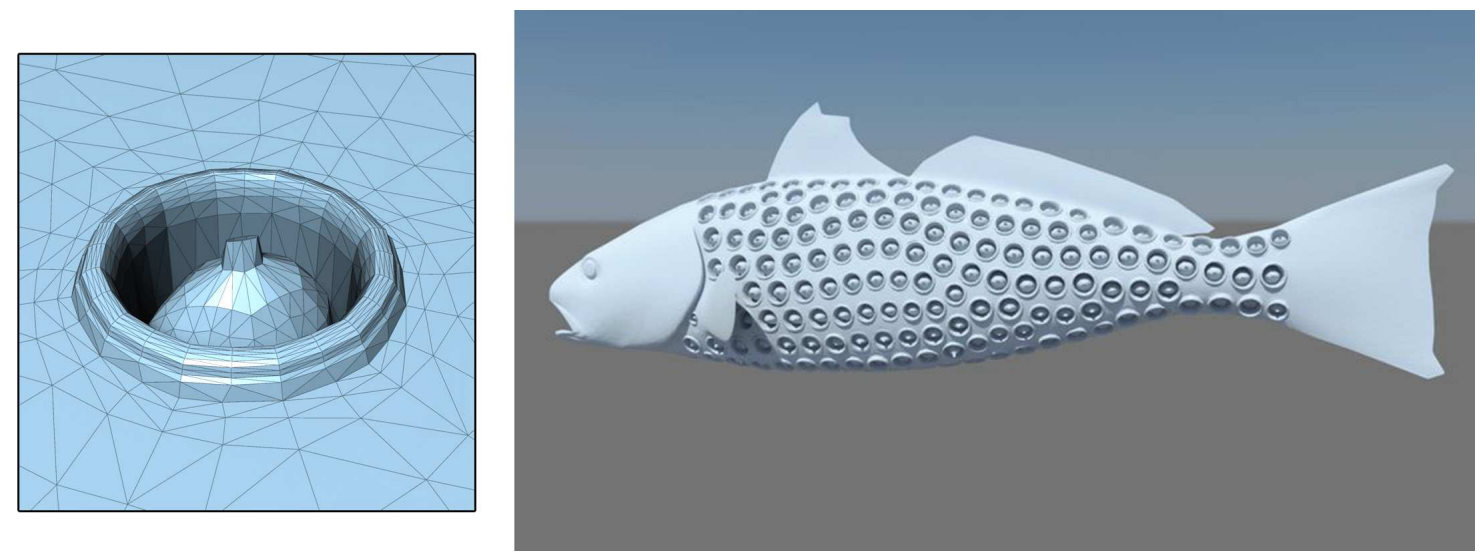

Fig. 30. A test of scales growing inward, under the surface, rather than outward. Left - the proxy model used to create the surface.

We perform another test with an unusual proxy model shape in Figure 30. We show that our scales do not necessarily need to protrude outward from a surface. In this case, the proxy model embeds into the surface of the mesh. The scale deformation has no restriction on the direction a scale emerges from the mesh, and handles this case well.

In Figure 31, an artist colored the forehead as a scale generation region and drew a lateral line up the middle of the forehead. Using the 4-pronged proxy model provided by the user, our method grew scales up the forehead of the model. We then performed Loop subdivision [Loop 1987] on this surface to demonstrate the water-tight nature of our surfaces.

Figure 32 shows a practical use of our scale synthesis in modeling a Stegosaurus. Beginning with the basic model, we wish to add spinal plates and tail spikes to the animal. First, we paint the region along the spine to grow scales. Because the spinal 

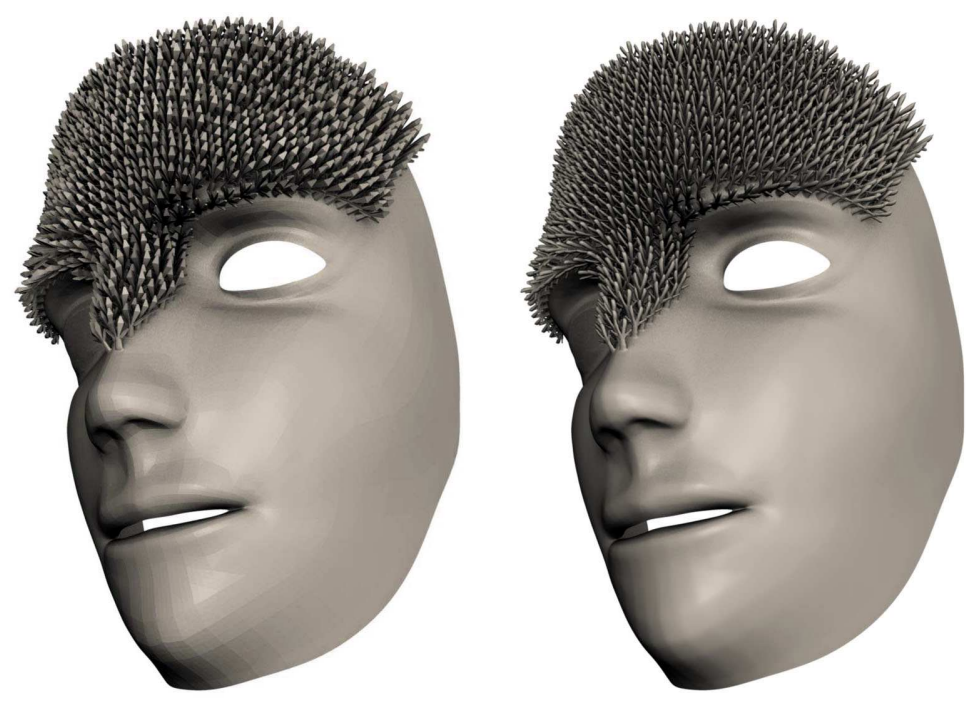

Fig. 31. Left - A face with a four-pronged scale applied. Right - the same model with Loop subdivision applied. Model credit: Jessica Riewe.

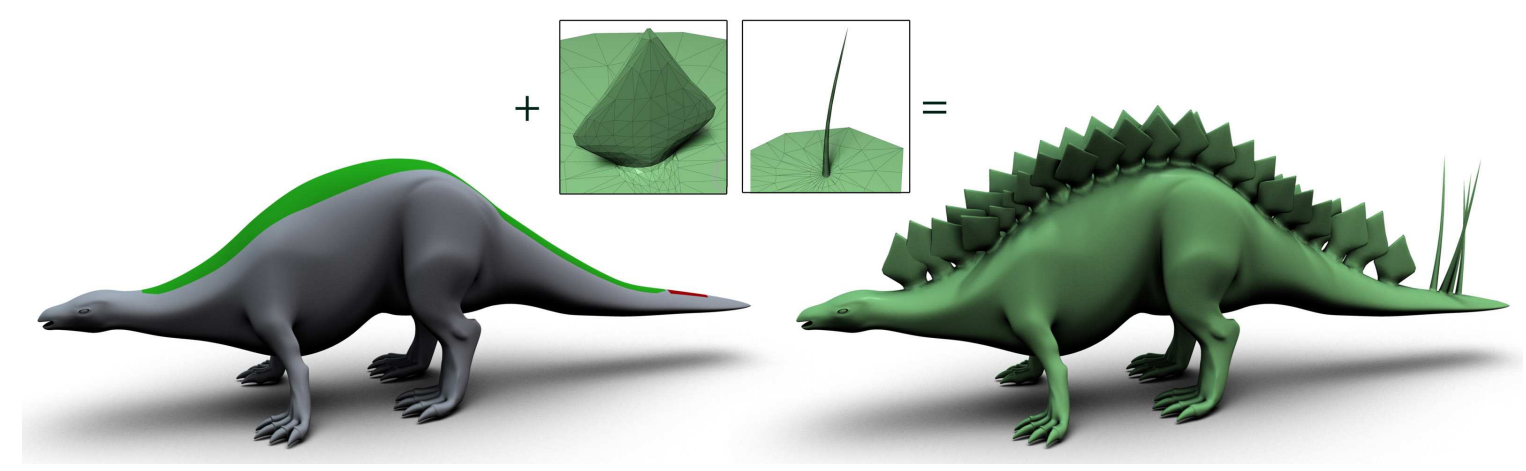

Fig. 32. Left A stegosaurus model, without back plates or tail spikes. The back plate growth region is colored green, and the tail spike region is colored red. Middle proxy models for the plates and spikes. Right - the stegosaurus after plates and spikes have been added. 
plates are rather large surface features, we seed a small number of scales to obtain large Voronoi regions. After growing the spinal plates, we then re-paint the resulting model's tail and grow 4 spine-shaped scales. The resulting model is recognizable as a Stegosaurus, and shows the usefulness of our technique even for large features. This model also illustrates the usefulness of having control over anisotropy in scale regions. Notice that the tail spikes of the stegosaurus have an isotropic shape at their base, while, the plates are anisotropic. Using more anisotropic Voronoi regions for the plates lets them fit their regions better, reducing the boundary space between adjacent scales.

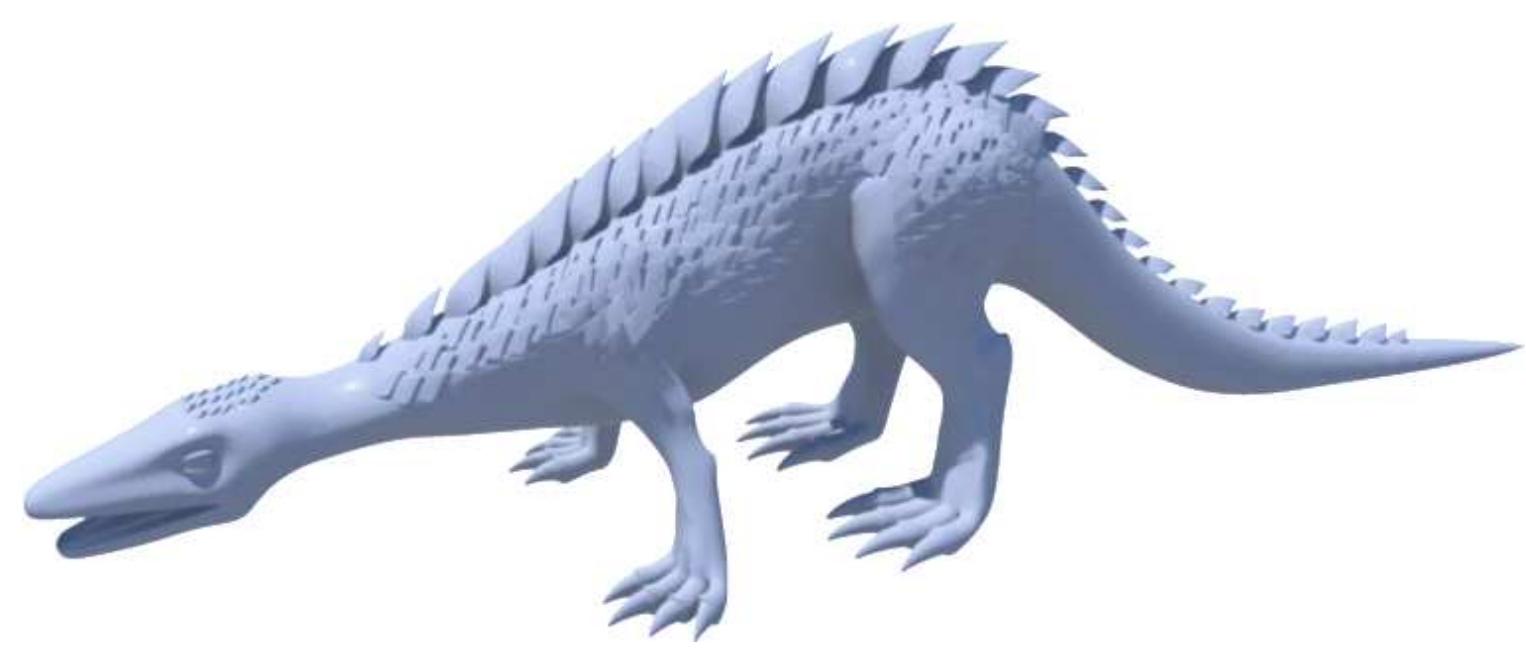

Fig. 33. A dragon with three types of scales applied to three different regions - the back, flank, and head.

We also test this multi-stage approach on a dragon model in Figure 33. In this case, we paint three different regions of the model, each time applying a different proxy model to that region. For the spine, we use a small number of sites with a thick, tall scale. The haunches utilize many flattened, rhombic scales. Third, we apply rounded, slightly keeled scales to the top of the head. The final model shows the successful integration of these scales into a single complex model. In this manner, 
an artist can iteratively paint the surface of a mesh with scale geometry.
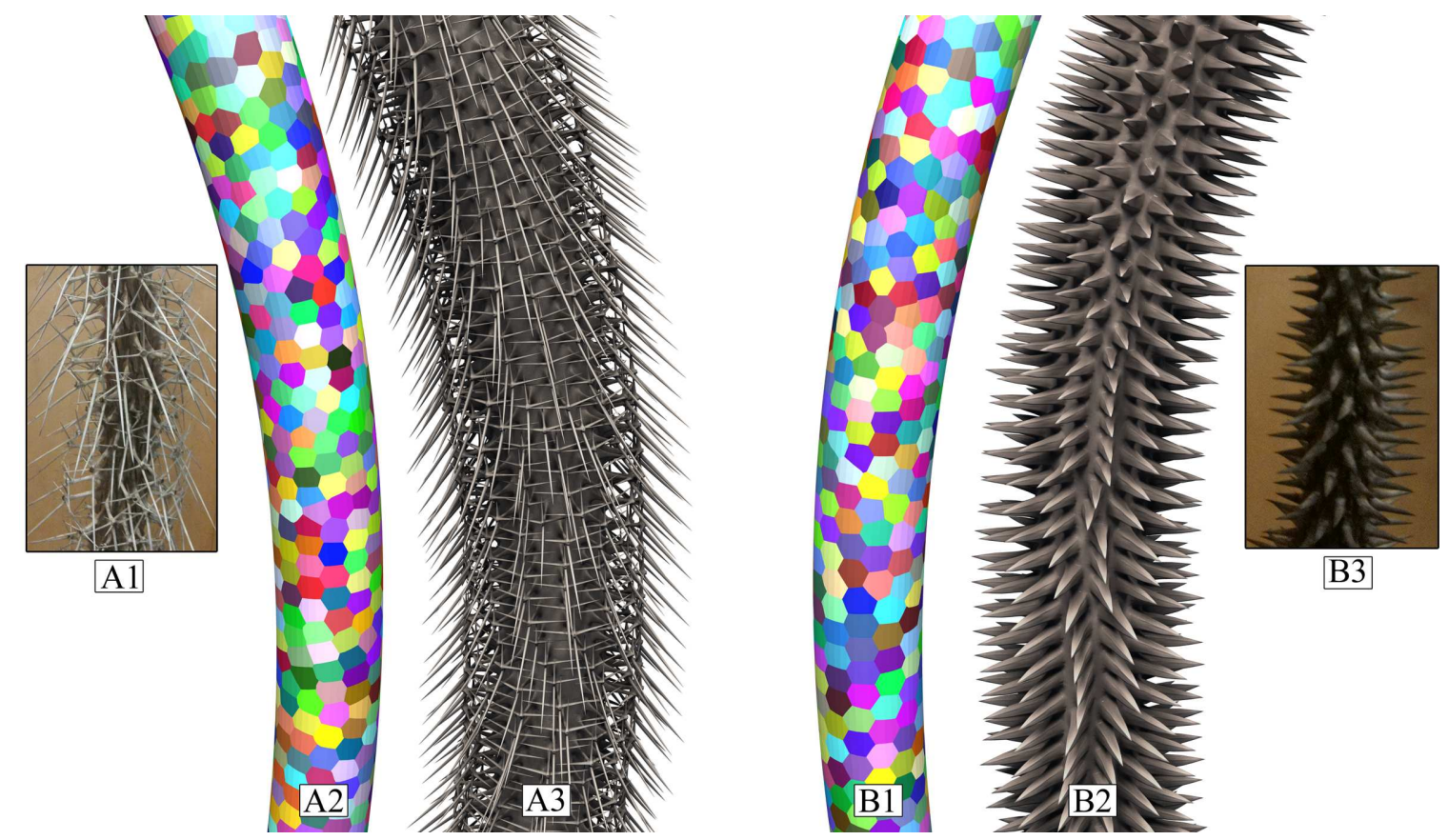

Fig. 34. A1, B3 - photographs of a Didiera madagascariensis and Alluaudia montagnacii, respectively. A2, B1 - CVTs from which thorn-scales were grown. A3, B2 - meshes of the plants with thorn-scales applied.

Our technique works well for creating thorns and thorn-like shapes such as those shown in Figure 34. Even complex thorn shapes, such as the 4-spiked thorn of the Didiera madagascariensis plant, pose no problem for our method. In the figure it also becomes apparent that the plants in the photos have some irregularities due to growth and environmental figures, while the synthesized versions exhibit more regularity. We mention a possible way to address this issue in Chapter VII

We apply rhomboid scales to an egg shape to create a scaled dragon egg in Figure 35 . We then attach high quality shaders and lighting to the model, and render it alongside a version simplified using quadric error simplification. These examples show that not only can we produce high degrees of detail with little effort, but the 

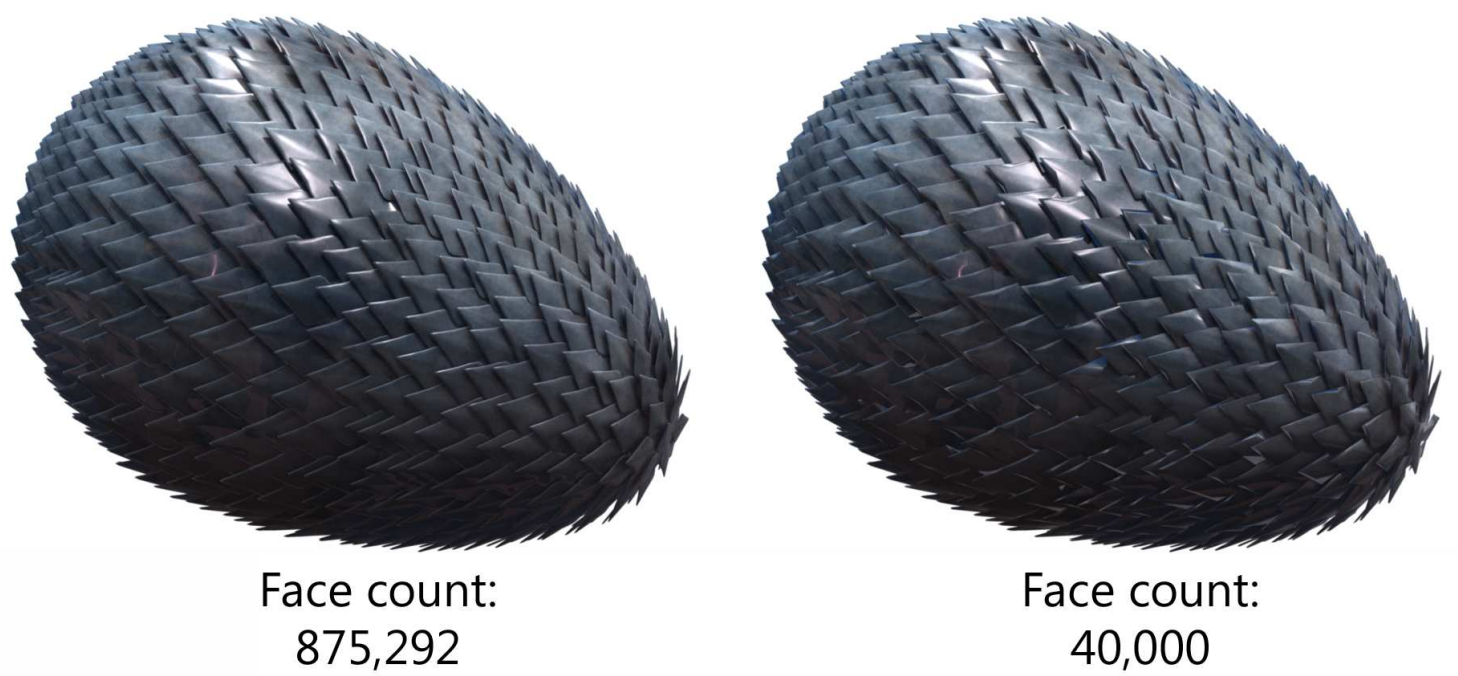

Fig. 35. Left - A dragon egg, covered in scales. Right - the same model, after surface simplification has been performed.

mesh renders well even when reduced to $1 / 20$ th of its original polygon count.

Our scale generation technique is useful for more than just animals and plants. As we noted in Chapter II, scales are used in many forms of artwork as well as in military armor. In Figure 36, we use our method to grow scales on a hauberk. Note that scale directions conform well to the surface, even at the shoulders.

Our approach has some limitations. Since we do not explicitly detect intersections between scales, it is possible that some scales may protrude into adjacent regions, especially in the presence of high amounts of negative curvature with respect to the size of the scale. Figure 37 shows an example of such intersection in the center of the saddle. Large positive curvature of the surface model such as high frequency ridges with respect to scale size can also cause issues because we only constrain the boundary of the scale to match the surface geometry. Laplacian deformation will tend to create something similar to a minimal surface in this situation instead of extrapolating the shape of the ridge. Nevertheless, in most situations, our method 


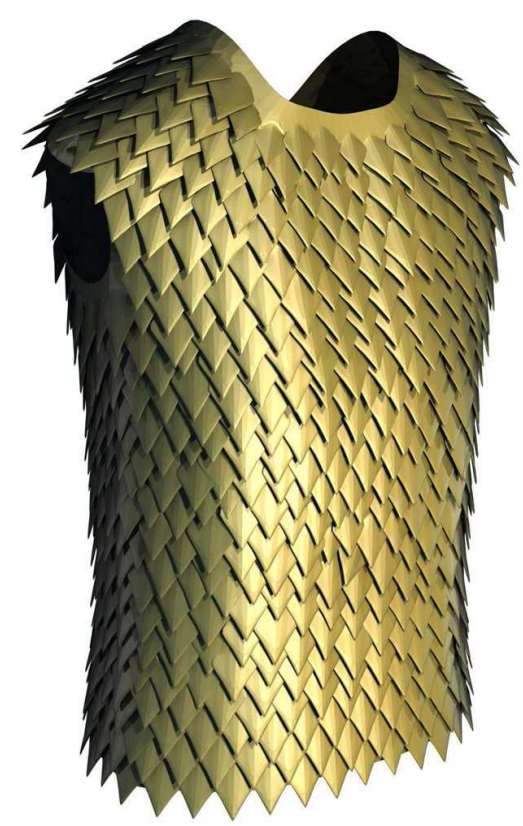

Fig. 36. A scale armored hauberk, showing scales generated by our technique.
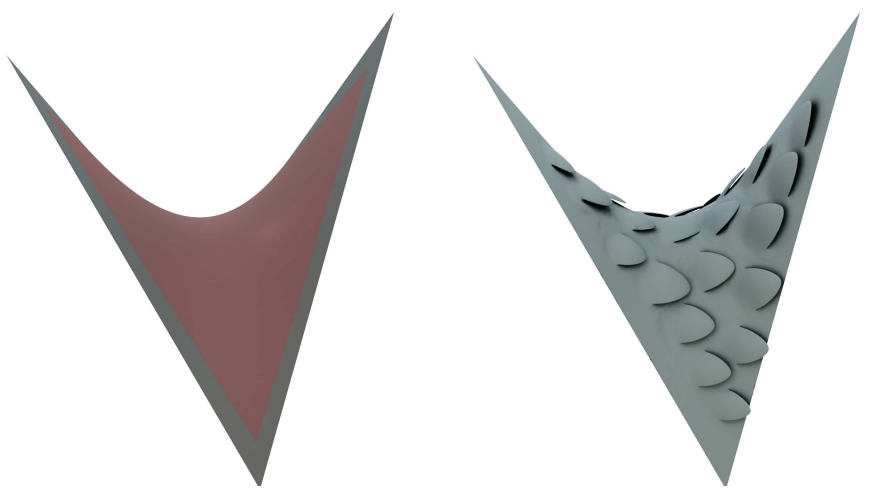

Fig. 37. A problem case for our method. High frequency details and regions of high negative curvature with respect to the scale size may cause self-intersection or twisting during scale deformation. 
tends to work well.

Our process takes a fairly long time to complete. In the case of a dense mesh like the flounder in Figure 26, completion took well over an hour on a Intel Core 2 Duo computer. The reason for this lengthy time lies both in geometric and algorithmic complexity. The flounder model for example contains 6.4 million polygons, due in large part to a proxy model with 1,800 faces being used for over 4,000 scales. As for algorithmic complexity, we found that the construction of the CVT and the final deformation of each scale dominate the running time. In the case of the CVT, the complexity of each Lloyd iteration is $(O(m \log n))$ where $m$ is the number of vertices and $n$ is the number of Voronoi sites, and running to convergence may take as many as 1000 steps. While the computation time of Lloyd's algorithm varies based on the complexity of the mesh, number of sites, and initial configuration of sites, performing 1000 iterations took from thirty minutes to an hour for most of our examples, again computed on an Intel Core 2 Duo processor.

For the Laplacian-based deformation, we construct a very large sparse matrix. The dimensionality of this matrix depends on the complexity of the proxy model, and in some cases exceeded dimensions of $8000 \times 8000$. We use a conjugate gradient solver with 1500 iterations to invert this sparse matrix for each scale, a process which may take over a second. Fortunately, our process is vectorizable, as each scale is synthesized completely independent of others. Every scale can be created simultaneously, so the the computation time has a lower bound of the processing time of a single scale. This parallelization also alleviates many memory concerns, as we can store the mesh for each scale on disk as they are processed, rather than storing the entire mesh in memory. 


\section{CHAPTER VII}

\section{CONCLUSION}

\section{A. Contribution}

We have provided a novel surface-replacement technique that generates scales and scale-like structures on a mesh. Our technique gives an artist the ability to control the arrangement of scales using a single stroke. Our method automatically fills a region with oriented scales and generates a well connected surface from artist-created proxy models.

Creating these models with traditional techniques can be incredibly time consuming and very difficult. The polygon count of a scaled model will typically be high, upwards of a million polys. This is a necessary consequence as a single scale consisting of a thousand polys will be repeated thousands of times, leading to high numbers of polys if you wish to retain geometric detail on the surface. This high degree of geometric complexity makes them visually striking, but causes problems for interactive 3D modeling programs such as Autodesk's Maya software. In addition, thin overlapping shapes are particularly bothersome for artists to deal with in the modeling process. Therefore, an automated scale generation technique is not only useful, but required if we wish to have a well-formed geometric surface of scales.

Scale creation covers a neglected area in repetitive skin structures for graphics. The two most commonly studied skin structures, feathers and hair, do share some similarities with scales. Hair, scales, and feathers all form self-similar shapes embedded in the skin of an animal. The placement of these structures tend towards Poisson distributions, as do many other body parts such as retinal cells [Yellott 1983]. In fact, Poisson distributions occur often in nature, even at the atomic level. 
Unlike scales, feathers and hair form very small cross-sections with the skin at the penetration point. Feather quills and hair shafts form very small, almost pointlike intersections with the skin. In comparison to these small, isotropic intersections, scales cover a much larger area of skin and form anisotropic regions of influence. Also, hair and feather synthesis techniques do not typically integrate their structures into the skin, due to the point-like nature of the intersection. In contrast, scales integrate visibly with skin, and so topological connectivity becomes important.

The shape of scales also deviates from hair and feather shapes. Hairs form long filaments, thin structures with imperceptible surface area. Feathers form flat, sheetlike structures. Scales, on the other hand, form fully 3d structures. This distinction emphasizes the importance of developing surface synthesis techniques specially tailored for scales.

Creating geometric patterns on a surface by defining a region on a surface with simple artist-provided constraints, then synthesizing geometry, is a fairly new area of research. Surface geometry synthesis has recent developments by Takayama et al.s Geobrush[36] tool and vector displacement tools in Pixologics Zbrush software. As computer processing speeds increase, the complexity of 3D models will correspondingly increase, and automated surface synthesis tools will become necessary for modeling as artists move away from manipulating individual faces and vertices.

As mentioned in the introductory chapter, maintaining a topologically manifold shape is important for architectural structures, including scale-like sculptures made by Peter Randall-Page [Randall-Page 2006] and Simon Thomas [Thomas 2006]. Volumetric shapes such as Bhat et al.'s textures [Bhat et al. 2004] are unsuitable for most architectural applications. Many construction techniques require a manifold surface made of connected panels to produce a lightweight, sturdy structure, so by maintaining this manifold topology, our method produce shapes which can be used 
for creating real sculptures.

B. Future Work

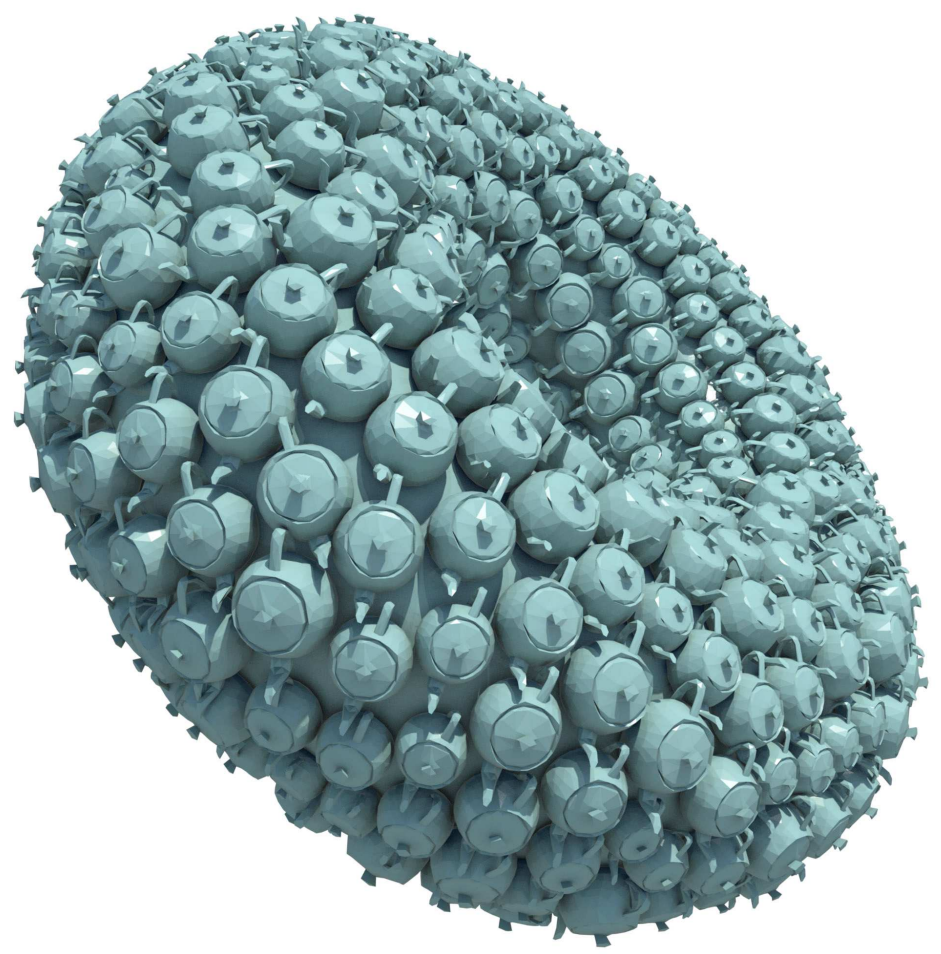

Fig. 38. A torus with teapot-shaped scales applied. Our method is robust enough to handle unusual shapes such as these.

The work in this dissertation is designed with a flexible framework in mind, which imposes few restrictions on the artist as far as the proxy models shape. The variety of scale shapes shown in the results reflects this robustness, even as far as allowing teapot-shaped scales as shown in Figure 38. 


\section{Scale Shape}

For example, the cycloid scales of bony fishes have some characteristics in common. The scales tend to have grooves radiating from the center of the scale, and also have growth rings which increase with a fishs age. In juvenile fish the scales are submerged under the skin, and emerge during maturation, then continue growing throughout the fishs life. Possible future work could automatically generate a proxy model, adjusting for variance in scale emergence, ring growth and grooves.

Another issue, related to parameterization of scale synthesis, is scale variation. In our work, the scales are identical to their proxy models, tending to create patterns that are more regular than natural objects. An example of this issue shows in Figure 34, where the photographed vine exhibits growth irregularities. While natural scales have self-similarity, they also slightly change across the surface of an object. To introduce variation into scales, we need a parametric model to morph scales over the surface. The user could constrain this morphing by specifying a scale shape at certain Voronoi regions on the surface, and then smoothly blend between the shapes on the surface.

Because we do not handle self-intersection, one extension of our work would involve collision testing for scales. Possible solutions might include a robust deformation which constrains scale synthesis to non-intersecting regions.

\section{Scale Arrangement}

In addition to further exploration of controlling the shape of scales, many options present themselves for improving squamations. One apparent improvement would be introduction of anisotropy in the distribution of the scales. While our work presents a method for creating anisotropy in the shape of scale regions, these regions will tend to cover the same surface area. In some animals, particularly reptiles, scale sizes 
vary across different body regions. The topic of altering Lloyd's algorithm to create different sized regions has been explored thoroughly for dithering applications, using techniques such as capacity-constrained CVT's [Balzer et al. 2009; Li et al. 2009]. Combining such a method with some form of artist control over region sizes would improve the realism of scales and their squamation.

Shape matching synthetic squamations to those of real animals is also a plausible extension of our work. By developing some form of image analysis which detects scale positions or patterns such as flow lines, we could constrain or even replace Lloyd's algorithm as a method for positioning scales. Statistical analysis of data obtained from real animals could also guide scale positions by estimating a 'realism' quality of scale arrangements and guiding scales towards arrangements having a higher quality metric.

\section{Texturing and Shading}

Another concern with generating a complex scaled surface is texturing or painting the surface. This dissertation creates the geometric shape of scales, producing manifold topology suitable for texturing. However, while our framework is not based upon a 2D parameterization, we would need to develop such a parameterization to perform $2 \mathrm{D}$ texturing. A possible solution could involve parameterizing and texturing the proxy model itself. Because the proxy model is cut and stitched with others, this process would introduce seams along scale boundaries. However, recent advancements in

content-aware image synthesis [Barnes et al. 2009] could be applied to correct the seams.

Shading of scales is another significant area of potential work. Animal and plant scales have a variety of reflective properties, and representation of these properties would be a useful area of study. Many animal scales are covered with a very thin, 
semi-transparent layer of epidermal skin. The scale core may consist of translucent materials such as keratin or guanine. Many fish scales contain exotic reflecting structures such as flattened photonic crystals, which Levy-Lior et al. recently explored [Levy-Lior et al. 2008]. Additionally, most animal species have pigmentation patterns over their bodies, and many of these patterns repeat over scales. From these observations, developing reflectance models for even a single species' scales is an interesting and nontrivial task. 


\section{REFERENCES}

Andersen, V., Desbrun, M., Bærentzen, J. A., And Aanæs, H. 2009. Height and tilt geometric texture. In $I S V C$ '09. Springer-Verlag, Berlin, Heidelberg, 656667.

Balzer, M., Schlömer, T., And Deussen, O. 2009. Capacity-constrained point distributions: a variant of lloyd's method. ACM Trans. Graph. 28, 86:1-86:8.

Bangay, S. 2007. Animated feather coats using field lines. In Proceedings of the 5th International Conference on Computer Graphics, Virtual Reality, Visualisation and Interaction in Africa. New York, 169-176.

Barnes, C., Shechtman, E., Finkelstein, A., And Goldman, D. B. 2009. Patchmatch: a randomized correspondence algorithm for structural image editing. ACM Trans. Graph. 28, 24:1-24:11.

Bhat, P., Ingram, S., And Turk, G. 2004. Geometric texture synthesis by example. In Proceedings of the Symposium on Geometry Processing. 41-44.

Bishop, M. And Coulston, J. 1993. Roman Military Equipment: From the Punic Wars to the Fall of Rome. Batsford.

Bloomenthal, J. 1985. Modeling the mighty maple. In Proceedings of the 12th Annual Conference on Computer Graphics and Interactive Techniques. New York, $305-311$.

Breder, C. M. 1947. An analysis of the geometry of symmetry with especial reference to the squamation of fishes. Bulletin of the American Museum of Natural History 88, 6, 325-412. 
Brown, M. E., Ed. 1957. The Physiology of Fishes. Academic Press Inc., New York.

Chen, Y., Xu, Y., Guo, B., And Shum, H.-Y. 2002. Modeling and rendering of realistic feathers. In Proceedings of SIGGRAPH. 630-636.

Cichorius, C. 1896. Die Reliefs der Traianssaule. Verlag von Georg Reimer, Berlin.

Cohen-Steiner, D., Alliez, P., And Desbrun, M. 2004. Variational shape approximation. In Proceedings of SIGGRAPH. 905-914.

Cook, R. L. 1984. Shade trees. In Proceedings of SIGGRAPH. 223-231.

Cornelius, G. 2005. Roman scale armour detail. http://commons.wikimedia. org/wiki/File:Roman_scale_armour_detail.JPG.

Dai, W.-K., Shih, Z.-C., And Chang, R.-C. 1995. Synthesizing feather textures in galliformes. Comput. Graph. Forum 14, 3, 407-420.

DeERING, M. F. 2005. A photon accurate model of the human eye. In Proceedings of SIGGRAPH. 649-658.

Demko, S., Hodges, L., And Naylor, B. 1985. Construction of fractal objects with iterated function systems. In Proceedings of the 12th Annual Conference on Computer Graphics and Interactive Techniques. New York, 271-278.

Denny, W. B. 1974. Blue-and-white islamic pottery on chinese themes. Boston Museum Bulletin 72, 368, 76-99.

Du, Q., Faber, V., And Gunzburger, M. 1999. Centroidal voronoi tesselations: applications and algorithms. SIAM Review 41, 4, 637-676.

Du, Q., Gunzburger, M. D., And Ju, L. 2002. Constrained centroidal voronoi tessellations for surfaces. SIAM J. Sci. Comput. 24, 5, 1488-1506. 
Du, Q. And WAng, D. 2005. Anisotropic centroidal voronoi tessellations and their applications. SIAM J. Sci. Comput. 26, 3, 737-761.

Fisher, M., Schröder, P., Desbrun, M., And Hoppe, H. 2007. Design of tangent vector fields. ACM Trans. Graph. 26.

Fleischer, K. W., Laidlaw, D. H., Currin, B. L., and Barr, A. H. 1995. Cellular texture generation. In Proceedings of the 22nd Annual Conference on Computer Graphics and Interactive Techniques. New York, 239-248.

Garland, M. And Heckbert, P. S. 1997. Surface simplification using quadric error metrics. In Proceedings of the 24th Annual Conference on Computer Graphics and Interactive Techniques. New York, 209-216.

Hart, J. C. And BAKer, B. 1996. Implicit modeling of tree surfaces. In Proceedings of Implicit Surfaces. 143-152.

Herodotus. 1954. The Histories. Penguin Books, New York.

HONDA, H. 1971. Description of the form of trees by the parameters of the tree-like body: Effects of the branching angle and the branch length on the shape of the tree-like body. Theoretical Biology 31, 331-338.

Itoh, T., Miyata, K., And Shimada, K. 2003. Generating organic textures with controlled anisotropy and directionality. IEEE Computer Graphics and Applications 23, 38-45.

Kardong, K. V. 1998. Vertebrates: Comparative Anatomy, Function, Evolution. McGraw-Hill.

Laufer, B. 1914. Chinese Clay Figures. Vol. 13. Chicago:Field Museum of Natural History. 
LDOT. 2007. Issyk's gold cataphract warrior. http://commons.wikimedia.org/ wiki/File:Issyk_Golden_Cataphract_Warrior.jpg.

LÉvy, B. AND LiU, Y. 2010. Lp centroidal voronoi tessellation and its applications. ACM Trans. Graph. 29, 119:1-119:11.

Levy-Lior, A., Pokroy, B., Levavi-Sivan, B., Leiserowitz, L., Weiner, S., AND ADDADI, L. 2008. Biogenic guanine crystals from the skin of fish may be designed to enhance light reflectance. Crystal Growth \& Design 8, 2, 507-511.

Li, H., Nehaby, D., Weiy, L.-Y., Sander, P. V., and Fuz, C.-W. 2009. Fast capacity constrained voronoi tessellation. In Proceedings of the 2009 Symposium on Interactive 3D Graphics and Games. New York, 13:1.

Liu, L., Zhang, L., Xu, Y., Gotsman, C., And Gortler, S. J. 2008. A local/global approach to mesh parameterization. Computer Graphic Forum 27, 5, $1495-1504$.

Liu, Y., Wang, W., Lévy, B., Sun, F., Yan, D.-M., Lu, L., and Yang, C. 2009. On centroidal voronoi tessellation - energy smoothness and fast computation. ACM Trans. Graph. 28, 101:1-101:17.

LLOYD, S. P. 1982. Least squares quantization in PCM. IEEE Transactions on Information Theory IT-28, 2, 129-137.

Loop, C. 1987. Smooth subdivision surfaces based on triangles. M.S. thesis, University of Utah, Utah.

Meskkauskas, A., McNulty, L. J., And Moore, D. 2004. Concerted regulation of all hyphal tips generates fungal fruit body structures: experiments with 
computer visualizations produced by a new mathematical model of hyphal growth. Mycological Research 108, 4, 341-353.

NOAA. 2011. National oceanic and atmospheric administration photo library. http://www.photolib.noaa.gov.

Palacios, J. And Zhang, E. 2007. Rotational symmetry field design on surfaces. ACM Trans. Graph. 26.

Palubicki, W., Horel, K., Longay, S., Runions, A., Lane, B., Měch, R., And Prusinkiewicz, P. 2009. Self-organizing tree models for image synthesis. ACM Trans. Graph. 28, 58:1-58:10.

Patterson, R. T., Wright, C., Chang, A. S., Taylor, L. A., Lyons, P. D., Dallimore, A., And Kumar, A. 2002. Atlas of common squamatological (fish scale) material in coastal british columbia, and an assessment of the utility of various scale types in paleofisheries reconstruction. Palaeontologia Electronica 4 , $1-88$.

Peng, J., Kristuansson, D., And Zorin, D. 2004. Interactive modeling of topologically complex geometric detail. In Proceedings of SIGGRAPH. 635-643.

Pinkall, U., Juni, S. D., And Polthier, K. 1993. Computing discrete minimal surfaces and their conjugates. Experimental Mathematics 2, 1, 15-36.

Porumbescu, S. D., Budge, B., Feng, L., And Joy, K. I. 2005. Shell maps. ACM Trans. Graph. 24, 3, 626-633.

Praun, E., Finkelstein, A., And Hoppe, H. 2000. Lapped textures. In Proceedings of SIGGRAPH. 465-470. 
Prusinkiewicz, P., Lindenmayer, A., And Hanan, J. 1988. Development models of herbaceous plants for computer imagery purposes. In Proceedings of the 15th Annual Conference on Computer Graphics and Interactive Techniques. New York, 141-150.

Radinsky, L. B. 1987. The Evolution of Vertebrate Design. The University of Chicago Press.

Randall-Page, P. 2006. Collaboration on the integration of sculptures and architecture in the eden project. In Bridges London Conference Proceedings. 1-12.

Ray, N., Vallet, B., Li, W. C., And LÉvy, B. 2008. N-symmetry direction field design. ACM Trans. Graph. 27, 10:1-10:13.

Rong, C. 1244. Nine dragons. Museum of Fine Arts, Boston.

Runions, A., Fuhrer, M., Lane, B., Federl, P., Rolland-Lagan, A.-G., AND Prusinkiewicz, P. 2005. Modeling and visualization of leaf venation patterns. ACM Trans. Graph. 24, 702-711.

Segraves, J. 2007. Assets / \#64102: Nine-banded armadillo. http://library . byways.org/assets/64102.

Sharf, A., Blumenkrants, M., Shamir, A., And Cohen-Or, D. 2006. Snappaste: an interactive technique for easy mesh composition. Vis. Comput. 22, 9, 835-844.

Sire, J.-Y. AND ARnulf, I. 1990. The development of squamation in four teleostean fishes with a survey of the literature. Ichthyological Research 37, 2, 133-143. 
Sorkine, O., Cohen-Or, D., Lipman, Y., Alexa, M., Rössl, C., And SeiDEL, H.-P. 2004. Laplacian surface editing. In Proceedings of the Symposium on Geometry Processing. 175-184.

Streit, L. M. 2004. Modelling of feather coat morphogenesis for computer graphics. Ph.D. thesis, University of British Columbia.

Streit, L. M. AND Heidrich, W. 2001. Modelling the embryological distribution of follicles. In In Proceedings of Western Computer Graphics Symposium (SKIGRAPH 01. 41-47.

Takayama, K., Schmidt, R., Singh, K., Igarashi, T., Boubekeur, T., And Sorkine, O. 2011. Geobrush: Interactive mesh geometry cloning. Computer Graphics Forum 30, 2, to appear.

Teichmann, M. And Teller, S. Polygonal approximation of voronoi diagrams of a set of triangles in three dimensions. Tech. Rep. 766, Lab of Comp. Sci., MIT.

Thomas, S. 2006. Love, understanding and soap bubble. In Bridges London Conference Proceedings. 33-40.

UlAm, S. 1962. On some mathematical properties connected with patterns of growth of figures. In Proceedings of Symposia on Applied Mathematics. American Mathematical Society, 215-224.

U.S. Army. 2010. File:peo soldier dragonskin.jpg. http://en.wikipedia.org/ wiki/File:PEO_soldier_Dragonskin.jpg.

Wang, I. R., Wan, J. W. L., And Baranoski, G. V. G. 2004. Physicallybased simulation of plant leaf growth: Research articles. Comput. Animat. Virtual Worlds 15, 237-244. 
Wang, X., Wang, L., Liu, L., Hu, S., And Guo, B. 2003. Interactive modeling of tree bark. In Proceedings of the 11th Pacific Conference on Computer Graphics and Applications. Washington, D.C., 83-90.

Ward, K., Bertails, F., Kim, T.-Y., Marschner, S. R., Cani, M.-P., And Lin, M. C. 2007. A survey on hair modeling: Styling, simulation, and rendering. IEEE Transactions on Visualization and Computer Graphics 13, 213-234.

Weber, A. J. And Gornowicz, G. 2009. Collision-free construction of animated feathers using implicit constraint surfaces. ACM Trans. Graph. 28, 12:1-12:8.

Wu, Y., Kalra, P., And Magnenat-Thalmann, N. 1997. Physically-based wrinkle simulation and skin rendering. In Proceedings of the Eurographics Workshop on Computer Animation and Simulation. 69-79.

Xu, K., Cohne-Or, D., Ju, T., Liu, L., Zhang, H., Zhou, S., and Xiong, Y. 2009. Feature-aligned shape texturing. Proceedings SIGGRAPH Asia 2009, ACM Transactions on Graphics 28, 5, 108:1-108:7.

YellotT, J. I. 1983. Spectral consequences of photoreceptor sampling in the rhesus retina. Science 221, 4608, 382-385.

Ying, L., Hertzmann, A., Biermann, H., And Zorin, D. 2001. Texture and shape synthesis on surfaces. In Proceedings of the Eurographics Workshop on Rendering Techniques. 301-312.

Zhang, E., Mischaikow, K., And Turk, G. 2006. Vector field design on surfaces. ACM Trans. Graph. 25, 4, 1294-1326. 
Zhou, K., Huang, X., Wang, X., Tong, Y., Desbrun, M., Guo, B., And Shum, H.-Y. 2006. Mesh quilting for geometric texture synthesis. In Proceedings of SIGGRAPH. 690-697.

Zou, H. And Niswander, L. 1996. Requirement for bmp signaling in interdigital apoptosis and scale formation. Science 272, 5262, 738-741. 


\section{VITA}

Name

Address

Email Address

Education
Eric Benjamin Landreneau

Department of Computer Science and Engineering Texas A\&M University

TAMU 3112

College Station, TX 77843-3112

eland@tamu.edu

B.S., Computer Science, Texas A\&M University, 2001

M.S., Computer Science, Texas A\&M University, 2005

PhD., Computer Science, Texas A\&M University, 2011 